\title{
缺陷型石墨相氮化碳光催化剂的制备与应用研究进展
}

叶珊珊 ${ }^{1,2 \dagger}$, 冯程洋 ${ }^{1,2 \dagger}$, 王佳佳 ${ }^{1,2^{*}}$, 汤琳 ${ }^{1,2^{*}}$

1. 湖南大学环境科学与工程学院, 长沙 410082;

2. 湖南大学环境生物学与污染控制教育部重点实验室, 长沙 410082

$\dagger$ 同等贡献

*联系人, E-mail: wangjiajia07@hnu.edu.cn; tanglin@hnu.edu.cn

2020-12-29 收稿, 2021-02-17 修回, 2021-02-24 接受, 2021-04-14 网络版发表

湖南省创新型省份建设专项(2019RS3012)资助

摘要 半导体光催化剂是解决环境污染和能源危机的有效途径之一. 石墨相氮化碳 $\left(\mathrm{g}-\mathrm{C}_{3} \mathrm{~N}_{4}\right)$ 作为一种新兴的高效 催化剂, 具有较好的稳定性, 在光催化技术中展现出巨大的工程应用潜力. 然而, 未经改性的 $\mathrm{g}-\mathrm{C}_{3} \mathrm{~N}_{4}$ 可见光响应范 围有限, 并且光激发电荷载流子复合速率高, 从而导致光催化活性较低. 通过向 $\mathrm{g}_{-} \mathrm{C}_{3} \mathrm{~N}_{4}$ 中引入缺陷, 可以扩展光响 应区域, 并作为电子空穴激发的活性中心, 提高光催化性能. 本文在实验和理论研究进展的基础上, 系统地综述了 缺陷 $\mathrm{g}-\mathrm{C}_{3} \mathrm{~N}_{4}$ 的合成方法、缺陷位点对 $\mathrm{g}-\mathrm{C}_{3} \mathrm{~N}_{4}$ 的影响以及其在水处理中的应用, 如抗生素、有机农药的降解及降低 重金属毒性等处理方面, 还有在水分解、二氧化碳转化及光催化脱氮上的应用。最后, 针对缺陷 $\mathrm{g}-\mathrm{C}_{3} \mathrm{~N}_{4}$ 应用所面临 的挑战, 本文从机理探索和材料开发两方面提出了展望.

关键词 $\mathrm{g}-\mathrm{C}_{3} \mathrm{~N}_{4}$, 氮缺陷, 光催化, 电子空穴对, 能带调控

伴随着工农业的发展，环境污染和能源短缺问题 日益严峻.一方面, 经济的飞速发展破坏了环境, 造成 了不同程度的水污染、大气污染和固体废物污染 ${ }^{[1]}$. 另 一方面, 从能源角度来看, 地球上的煤炭、石油及天然 气等资源有限, 能源消耗逐步增长, 能源短缺问题会日 益严峻 ${ }^{[2]}$. 近年来, 太阳能作为新兴能源引起了研究人 员的广泛关注 ${ }^{[3 \sim 12]}$. 1972年, Fujishima和Honda ${ }^{[6]}$ 首次提 出光催化氧化技术, 指出了二氧化钛能在光照条件下 进行光解水. 之后, Pruden和Ollis ${ }^{[13]}$ 发现光催化技术在 水和大气污染物治理领域是一种极具潜力的处理办法. 在外界光源的照射下, 半导体材料将光能转化成化学 能, 进而实现能源转化和污染物去除, 光催化技术在解 决环境污染和缓解能源短缺问题上具有良好的效果.

1996年, Teter和Hemley ${ }^{[14]}$ 发现石墨相氮化碳 $(\mathrm{g}$ -
$\mathrm{C}_{3} \mathrm{~N}_{4}$ ) 不仅具有优异的稳定性, 还能够实现可见光响应. Wang等人 ${ }^{[15]}$ 首次报道了 $\mathrm{g}-\mathrm{C}_{3} \mathrm{~N}_{4}$ 能够在可见光下进行光 解水产氢. 近年来, $\mathrm{g}-\mathrm{C}_{3} \mathrm{~N}_{4}$ 的研究取得巨大的进步. $\mathrm{g}-\mathrm{C}_{3} \mathrm{~N}_{4}$ 因其具有原料低廉、合成方法简单、环境友好 等优点，在光解水制氢 ${ }^{[16 ~ 20]}$ 、废水脱毒 ${ }^{[21,22]}$ 、选择性 氧化 ${ }^{[23 \sim 27]}$ 和 $\mathrm{CO}_{2}$ 还原 ${ }^{[28 ~ 33]}$ 、环境修复 ${ }^{[34 \sim 39]}$ 等方面被广 泛应用. 除此之外, g- $\mathrm{C}_{3} \mathrm{~N}_{4}$ 还具有优异的化学稳定性、 热稳定性和光稳定性 ${ }^{[40 \sim 43]}$. 但由于 $g-\mathrm{C}_{3} \mathrm{~N}_{4}$ 对全光谱响 应弱, 光生载流子复合率高, 导致其光催化活性较低, 限制了 $\mathrm{g}-\mathrm{C}_{3} \mathrm{~N}_{4}$ 的实际应用. 为了克服上述问题, 研究人 员使用了各种策略来提升 $\mathrm{g}-\mathrm{C}_{3} \mathrm{~N}_{4}$ 的光催化性能，如掺 杂原子 $\left(\right.$ 如 $\mathrm{C}^{[44]} 、 \mathrm{~B}^{[45]} 、 \mathrm{O}^{[46]} 、 \mathrm{~S}^{[47]} 、 \mathrm{P}^{[48]} 、 \mathrm{Br}^{[49]}$ 和 $\mathrm{Fe}^{[50]}$ )、引人氮缺陷、异质结复合 ${ }^{[51 \sim 55]}$ 或贵金属修 饰 ${ }^{[56-59]}$ 等. 
$\mathrm{Niu}$ 等人 ${ }^{[60]}$ 将氮缺陷引人到 $\mathrm{g}-\mathrm{C}_{3} \mathrm{~N}_{4}$ 框架中, 这些缺 陷可以操纵电子结构, 产生的中间隙态可作为带尾态, 能够与价带或导带发生重叠. 半导体的中间隙态可扩 展光响应, 并作为电子空穴激发的活性中心. 在 $\mathrm{g}-\mathrm{C}_{3} \mathrm{~N}_{4}$ 中引入缺陷, 可以提高 $\mathrm{g}-\mathrm{C}_{3} \mathrm{~N}_{4}$ 的光催化活性. 本文在实 验和理论研究的基础上, 综述了缺陷 $g-\mathrm{C}_{3} \mathrm{~N}_{4}$ 的合成方 法、缺陷位点对 $\mathrm{g}-\mathrm{C}_{3} \mathrm{~N}_{4}$ 的影响, 以及其在废水脱毒、 水分解、 $\mathrm{CO}_{2}$ 转化及光催化脱氮等领域的应用. 同时, 也对缺陷 $g-\mathrm{C}_{3} \mathrm{~N}_{4}$ 应用所面临的挑战进行了讨论.

\section{1 石墨相氮化碳的性质}

未改性的 $\mathrm{g}-\mathrm{C}_{3} \mathrm{~N}_{4}$ 为无规则颗粒状, 常温下不溶于 水, 耐酸耐碱, 正常情况下呈淡黄色. 微观上 $\mathrm{g}-\mathrm{C}_{3} \mathrm{~N}_{4}$ 为 平面二维片层结构，分子间作用力使层与层结合. $\mathrm{g}-\mathrm{C}_{3} \mathrm{~N}_{4}$ 的基本结构单元有两种，其中，图1(a)显示的 $\mathrm{g}$ $\mathrm{C}_{3} \mathrm{~N}_{4}$ 属于 $R 3 m$ 空间群, 以三嗪环 $\left(\mathrm{C}_{3} \mathrm{~N}_{3}\right)$ 为结构单元; 图 1 (b)显示的 $g-\mathrm{C}_{3} \mathrm{~N}_{4}$ 属于 $P 6 m 2$ 空间群, 以七嗪环 $\left(\mathrm{C}_{6} \mathrm{~N}_{7}\right)$ 为 结构单元 ${ }^{[61]}$. 在这两种结构中, $\mathrm{C}$ 原子和 $\mathrm{N}$ 原子均发生 $\mathrm{sp}^{2}$ 杂化, 形成一个高度离域的共轭体系. 理论上结构 单元为七嗪环 $\left(\mathrm{C}_{6} \mathrm{~N}_{7}\right)$ 的 $g-\mathrm{C}_{3} \mathrm{~N}_{4}$ 更稳定, 与结构单元为三 嗪环 $\left(\mathrm{C}_{3} \mathrm{~N}_{3}\right)$ 的 $g-\mathrm{C}_{3} \mathrm{~N}_{4}$ 相比, 其热力学能量更低, 二者的 差值约为 $30 \mathrm{~kJ} / \mathrm{mol}^{[62 ~ 65]}$. 在多数情况下, 通过一般方法 制备的 $g-\mathrm{C}_{3} \mathrm{~N}_{4}$ 结晶度较低, 为非晶状聚合物. 而一些特 殊方法制备的 $\mathrm{g}-\mathrm{C}_{3} \mathrm{~N}_{4}$ 结晶度较高，在高分辨透射电子 显微镜下能观察到明显的晶格条纹, 孔尺寸为 $26.45 \mathrm{~nm}$ 左右, 晶格间距为 $0.326 \mathrm{~nm}$ 左右 ${ }^{[66-68]}$. 理论上, $\mathrm{g}-\mathrm{C}_{3} \mathrm{~N}_{4}$ 的比表面积可达 $2500 \mathrm{~m}^{2} / \mathrm{g}$, 然而 $\mathrm{g}-\mathrm{C}_{3} \mathrm{~N}_{4}$ 的层与 层之间存在严重的堆叠, 实际比表面积远小于此 ${ }^{[69]}$.

$\mathrm{C}$ 和 $\mathrm{N}$ 为 $\mathrm{g}-\mathrm{C}_{3} \mathrm{~N}_{4}$ 的组成元素, 理论上其 $\mathrm{C} / \mathrm{N}$ 值为 0.75 ,
分子量为 92 . 但是反应条件不同, 所得 $g-\mathrm{C}_{3} \mathrm{~N}_{4}$ 的 $\mathrm{C} / \mathrm{N}$ 有 差异. 以三聚氭氯和三聚氭胺为原料, $\mathrm{Li}$ 等人 ${ }^{[70]}$ 通过高 温固相法得到 $\mathrm{C} / \mathrm{N}$ 值为 0.60 , 形态为具有空心结构的纳 米棒. Komatsu ${ }^{[71]}$ 以 $\mathrm{NH}_{4} \mathrm{SCN}$ 为原料通过高温固相法合 成 $\mathrm{C} / \mathrm{N}$ 值为 0.71 , 结晶度优良的 $g-\mathrm{C}_{3} \mathrm{~N}_{4}$ 结构单元为七嗪 环. 除此之外, $g-\mathrm{C}_{3} \mathrm{~N}_{4}$ 表面丰富的碱基 $(-\mathrm{NH}-$ 、 $=\mathrm{N}-、-\mathrm{NH}_{2}$ 和 $\left.-\mathrm{N}-\mathrm{C}=\right)$ 有利于通过静电相互作用 的化学吸附去除酸性有毒分子 ${ }^{[72]}$. 但 $g-\mathrm{C}_{3} \mathrm{~N}_{4}$ 具有疏水 性, 会使层间作用力弱, 对表面催化反应有负面影响 ${ }^{[73]}$. $\mathrm{g}-\mathrm{C}_{3} \mathrm{~N}_{4}$ 的禁带宽度约为 $2.7 \mathrm{eV}$, 以七嗪环为结构单 元构筑的模型, 其价带和导带电位分别为 1.27 和 $-1.42 \mathrm{~V}$ (vs. $\mathrm{Ag} / \mathrm{AgCl}, \mathrm{pH}$ 6.6 ${ }^{[74]}$. 块状 $\mathrm{g}_{-} \mathrm{C}_{3} \mathrm{~N}_{4}$ 可以吸收 可见光, 但对可见光的吸收存在一定的局限, 只对波长 小于 $450 \mathrm{~nm}$ 的可见光具有吸收作用. 在大于或等于禁 带宽度能量的光照射下, $\mathrm{g}-\mathrm{C}_{3} \mathrm{~N}_{4}$ 产生电子空穴对, 价带 电子 $\left(\mathrm{e}^{-}\right)$跃迁到导带上, 价带上产生空穴 $\left(\mathrm{h}^{+}\right)^{[75]}$. 跃迁到 导带上的电子表现出还原能力, 在价带上的空穴具有 氧化能力 ${ }^{[76]}$. 在对有机污染物进行消除时, $e^{-}$与 $g-C_{3} N_{4}$ 吸附的溶解氧作用生成超氧根负离子 $\left(\cdot \mathrm{O}_{2}{ }^{-}\right)^{[77]} ; \mathrm{h}^{+}$可以 将污染物直接氧化, 或者氧化 $\mathrm{OH}^{-}$和 $\mathrm{H}_{2} \mathrm{O}$ 形成差基自由 基 $(\cdot \mathrm{OH}) \cdot \mathrm{O}_{2}{ }^{-}$和. $\mathrm{OH}$ 可以将有机物氧化为 $\mathrm{CO}_{2}$ 和 $\mathrm{H}_{2} \mathrm{O}$.

本征缺陷对光催化反应非常重要, 当氮缺陷被引 人到 $\mathrm{g}-\mathrm{C}_{3} \mathrm{~N}_{4}$ 框架中, 这些缺陷可以操纵电子结构并可 能作为反应活性中心，提高量子效率 ${ }^{[78,79]}$. 此外, 由于 氮缺陷引人可以导致价带上移和导带下移，产生的中 间隙态与价带或导带发生重叠, 可以拓展半导体的光 响应区域, 并为电子、空穴的激发提供活性位点, 降低 载流子的复合损耗，有利于长波长光子的吸收和光致 电子的俘获 ${ }^{[80]}$. 缺陷 $\mathrm{g}-\mathrm{C}_{3} \mathrm{~N}_{4}$ 能够对可见光有良好的响

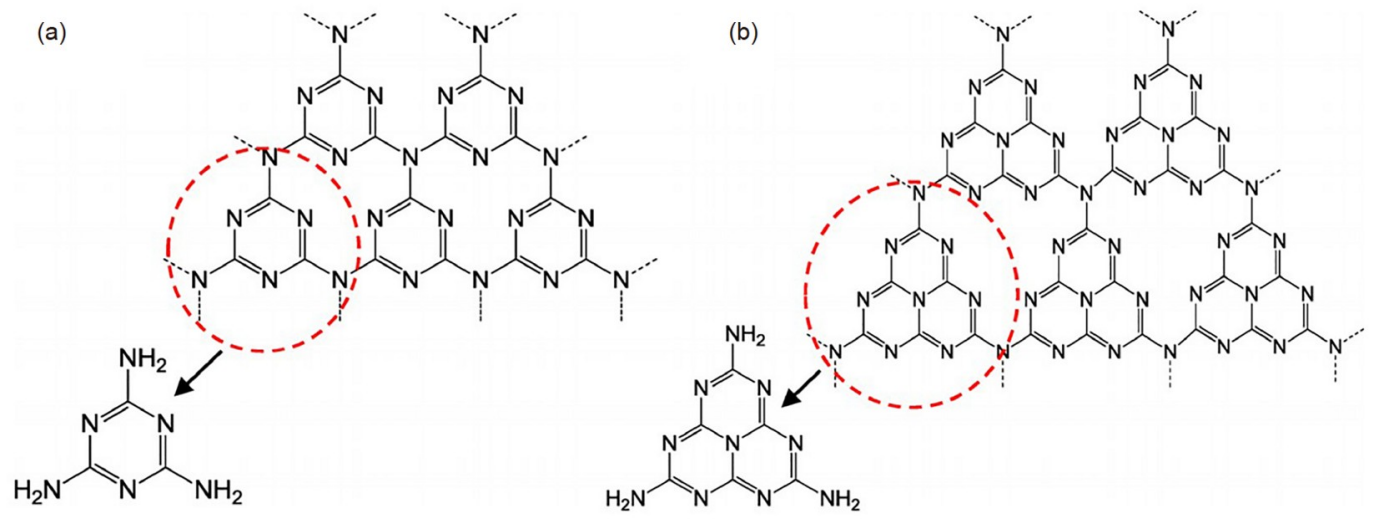

图 1 (网络版彩色) $\mathrm{g}-\mathrm{C}_{3} \mathrm{~N}_{4}$ 的三嗪环结构(a)和七嗪环结构(b) ${ }^{[15]}$

Figure 1 (Color online) Triazine ring structure of $\mathrm{g}-\mathrm{C}_{3} \mathrm{~N}_{4}$ (a) and hepazine ring structure of $\mathrm{g}_{-} \mathrm{C}_{3} \mathrm{~N}_{4}(\mathrm{~b})^{[15]}$ 
应, 且具有较大的比表面积, 光致载流子分离率显著提 高, 具有较高的析氢速率和优良的光催化稳定性 ${ }^{[81,82]}$. 将缺陷引人到 $g-C_{3} \mathrm{~N}_{4}$ 中是提高 $\mathrm{g}-\mathrm{C}_{3} \mathrm{~N}_{4}$ 催化活性的重要 方法, 可为制备高效 $g-\mathrm{C}_{3} \mathrm{~N}_{4}$ 光催化剂提供新思路 ${ }^{[83]}$.

\section{2 缺陷型石墨相氮化碳的制备方法}

g- $\mathrm{C}_{3} \mathrm{~N}_{4}$ 的合成原料十分丰富, 有双氰胺 $\left(\mathrm{C}_{2} \mathrm{H}_{4} \mathrm{~N}_{4}\right)$ 、 硫脲 $\left(\mathrm{CH}_{4} \mathrm{~N}_{2} \mathrm{~S}\right)$ 、尿素 $\left(\mathrm{CON}_{2} \mathrm{H}_{4}\right)$ 或三聚㲵胺 $\left(\mathrm{C}_{3} \mathrm{H}_{6} \mathrm{~N}_{6}\right)$ 等. 迄今用于合成缺陷 $\mathrm{g}-\mathrm{C}_{3} \mathrm{~N}_{4}$ 的方法都有其局限性, 限制 了引人氮缺陷的类型和丰度. 为了增强可见光响应能 力, 在 $\mathrm{g}-\mathrm{C}_{3} \mathrm{~N}_{4}$ 中引入氮缺陷是可行的操作. 本文将在 $\mathrm{g}-\mathrm{C}_{3} \mathrm{~N}_{4}$ 中引人缺陷的方法分为聚合前调节、聚合时调 节和聚合后调节3种方式来介绍. 聚合前调节是通过改 变前驱体来引入缺陷, 如在前驱体中添加氢氧化物、 嗍氢化钠等物质; 聚合时调节是在聚合时提供还原性 气氛, 如通人氢气、氨气等, 可以制备出含有不同氮空 位密度的 $g-\mathrm{C}_{3} \mathrm{~N}_{4}$; 聚合后调节是对合成的无缺陷 $g-\mathrm{C}_{3} \mathrm{~N}_{4}$ 进行改性，如进行再煅烧或者酸处理等以达到合成氮 空位的目的. 表 $\mathrm{S} 1$ 总结了通过不同途径向 $\mathrm{g}-\mathrm{C}_{3} \mathrm{~N}_{4}$ 中引 人缺陷的方法, 与原 $\mathrm{g}-\mathrm{C}_{3} \mathrm{~N}_{4}$ 相比, 所制备的缺陷 $\mathrm{g}-\mathrm{C}_{3} \mathrm{~N}_{4}$ 产氢速率都有不同程度的提高.

\section{1 聚合前调节}

聚合前进行调节, 可通过将 $\mathrm{g}-\mathrm{C}_{3} \mathrm{~N}_{4}$ 与其他物质, 如 $\mathrm{KOH} 、 \mathrm{NaOH} 、 \mathrm{NaBH}_{4}$ 或者硝酸等一起作为前驱体, 在一 定的条件下进行反应, 可以生成含有氮缺陷的 $g-\mathrm{C}_{3} \mathrm{~N}_{4}$.

$\mathrm{Yu}$ 等人 ${ }^{[84]}$ 介绍了一种以 $\mathrm{KOH}$ 辅助制备含氮缺陷 $\mathrm{g}-$ $\mathrm{C}_{3} \mathrm{~N}_{4}$ 的新方法, 该种方法能在尿素或其他富氮前体(如 三聚氰胺和硫艮)的热聚合过程中选择性地原位引人 氮缺陷, 如图2(a)所示. 在引入氮缺陷后, $g-\mathrm{C}_{3} \mathrm{~N}_{4}$ 的光吸 收范围边缘向红端方向发生移动, 结果表明氮缺陷浓 度对红移的大小有决定作用，而氮缺陷浓度可以通过 $\mathrm{KOH}$ :尿素的比例来调节. 此外, 掺杂的 $\mathrm{K}$ 离子不参与 缺陷 $\mathrm{g}-\mathrm{C}_{3} \mathrm{~N}_{4}$ 的电子结构, 但提高了其导电性, 有利于载 流子转移. 以碱辅助制备含氮缺陷的 $g-\mathrm{C}_{3} \mathrm{~N}_{4}$ 具有通用 性，因为使用其他碱性化合物(如 $\mathrm{NaOH}$ 和 $\left.\mathrm{Ba}(\mathrm{OH})_{2}\right)$ 也 可以获得目的产物. 由于增强了可见光吸收能力和改 善了载流子的分离效果, 缺陷 $\mathrm{g}-\mathrm{C}_{3} \mathrm{~N}_{4}$ 在可见光下表现 出的光催化性能远高于原始 $g-\mathrm{C}_{3} \mathrm{~N}_{4}$. 因此, 碱辅助合成 策略为优化 $g-\mathrm{C}_{3} \mathrm{~N}_{4}$ 基材料提供了一种简单有效的方法.

此外, 对 $g-\mathrm{C}_{3} \mathrm{~N}_{4}$ 的前驱体进行酸处理能够有效合 成缺陷 $\mathrm{g}-\mathrm{C}_{3} \mathrm{~N}_{4}$, 对前驱体进行酸聚缩合反应可得到三
聚氭胺 $-\mathrm{H}_{2} \mathrm{SO}_{4}$ 、三聚氰胺 $-\mathrm{CH}_{3} \mathrm{COOH}$ 和三聚氰胺$\mathrm{HNO}_{3}$. Wang等人 ${ }^{[85]}$ 酸化 $\mathrm{g}-\mathrm{C}_{3} \mathrm{~N}_{4}$ 的制备方法是: 将 $2 \mathrm{~g}$ 三 聚氰胺浸人 $5 \mathrm{~mL} 1 \mathrm{~mol} / \mathrm{L}$ 的 $\mathrm{HNO}_{3}$ 溶液中, 加人一定量 的乙醇, 室温下搅拌 $3 \mathrm{~h}$. 然后将混合物转移到 $100 \mathrm{~mL}$ 特氟龙密封的高压釜中, $180^{\circ} \mathrm{C}$ 保存 $12 \mathrm{~h}$, 冷却至室温 后，离心收集白色中间体，用水和乙醇洗涤至中性， $60^{\circ} \mathrm{C}$ 真空干燥过夜. 最后, 将得到的中间产物在氮气填 充的管式炉中 $550^{\circ} \mathrm{C}$ 下煅烧 $4 \mathrm{~h}$, 升温速率为 $2.3^{\circ} \mathrm{C} / \mathrm{min}$. 得到的缺陷 $\mathrm{g}-\mathrm{C}_{3} \mathrm{~N}_{4}$ 析氢活性高达 $8910.7 \mu \mathrm{mol} / \mathrm{g}$, 是 $\mathrm{g}-\mathrm{C}_{3} \mathrm{~N}_{4}$ 的9.9倍.

$\mathrm{Xu}$ 等人 ${ }^{[83]}$ 以乙酸处理过的三聚氰胺为前驱体，采 用热缩合法成功地将氮缺陷引入 $\mathrm{g}-\mathrm{C}_{3} \mathrm{~N}_{4}$ 中, 如图2(b)所 示. 将 $5.0 \mathrm{~g}$ 三聚氰胺分散于 $100 \mathrm{~mL}$ 乙酸中, 通过油浴加 热法, 将溶液加热至 $120^{\circ} \mathrm{C}$, 得到固体. 将得到的样品固 体放人有盖的氧化铝坩埚中, 然后以 $3^{\circ} \mathrm{C} / \mathrm{min}$ 的升温速 度, 在马弗炉中升温至 $550^{\circ} \mathrm{C}$ 并加热 $4 \mathrm{~h}$. 得到的固体在 玛瑙研钵中研磨, 得到的产物记为 CN-HAc. 醋酸与三 聚氧胺的结合对 $g-\mathrm{C}_{3} \mathrm{~N}_{4}$ 中氮缺陷的形成起着重要作用, 缺陷 $g-\mathrm{C}_{3} \mathrm{~N}_{4}$ 与原始 $\mathrm{g}-\mathrm{C}_{3} \mathrm{~N}_{4}$ 具有类似的晶体结构、比表 面积和粒径大小. 但 CN-HAc的可见光吸收范围扩大, 电荷分离和转移效率提升. CN-HAc的光催化析氢速率 稳定, 可达 $24 \mu \mathrm{mol} / \mathrm{h}$, 大约是原始 $\mathrm{g}-\mathrm{C}_{3} \mathrm{~N}_{4}$ 的 5 倍. 同时, $\mathrm{CN}-\mathrm{HAc}$ 对 RhB的光催化降解具有良好的活性, 对环境 修复具有重要意义.

\section{2 聚合时调节}

引人氮空位对提高 $\mathrm{g}-\mathrm{C}_{3} \mathrm{~N}_{4}$ 的光催化活性有积极作 用，但过多的氮空位会导致电子空穴对容易发生复 合 ${ }^{[86,87]}$. 目前, 控制 $\mathrm{g}-\mathrm{C}_{3} \mathrm{~N}_{4}$ 中氮空位的浓度是一个巨大 的挑战, 在聚合时提供一种还原性气氛是控制缺陷 g- $\mathrm{C}_{3} \mathrm{~N}_{4}$ 中氮空位浓度的方法之一.

Cui等人 ${ }^{[88]}$ 采用氨聚合法制备了缺陷g- $\mathrm{C}_{3} \mathrm{~N}_{4}$. 在氨 气气氛中, 一定量的硫氰酸铵 $\left(\mathrm{NH}_{4} \mathrm{SCN}\right)$ 在 $550^{\circ} \mathrm{C}$ 中加 热 $2 \mathrm{~h}$, 加热速率为 $5^{\circ} \mathrm{C} / \mathrm{min}$, 制得缺陷 $\mathrm{g}-\mathrm{C}_{3} \mathrm{~N}_{4}$. 与在氮气 气氛中制备的原始 $g-\mathrm{C}_{3} \mathrm{~N}_{4}$ 相比, 制得的样品具有较好 的分散片状形貌、表面积较大、介孔结构较多、结晶 度较高. 由于缺陷 $\mathrm{g}-\mathrm{C}_{3} \mathrm{~N}_{4}$ 活性位点分布更广, 光生载流 子在改进的有序微观结构中有更有效的运输和分离效 率, 在可见光照射下, 缺陷 $\mathrm{g}-\mathrm{C}_{3} \mathrm{~N}_{4}$ 的光催化析氢速率更 高, 约为原始 $\mathrm{g}-\mathrm{C}_{3} \mathrm{~N}_{4}$ 的6倍.

$\mathrm{Tu}$ 等人 ${ }^{[89]}$ 以三聚氭胺为原料, 在 $550^{\circ} \mathrm{C}$ 下热缩聚 $4 \mathrm{~h}$, 制备了 $\mathrm{g}-\mathrm{C}_{3} \mathrm{~N}_{4}$ 块体材料. 在氢气气氛下, 将 $\mathrm{g}-\mathrm{C}_{3} \mathrm{~N}_{4}$ 
(a)
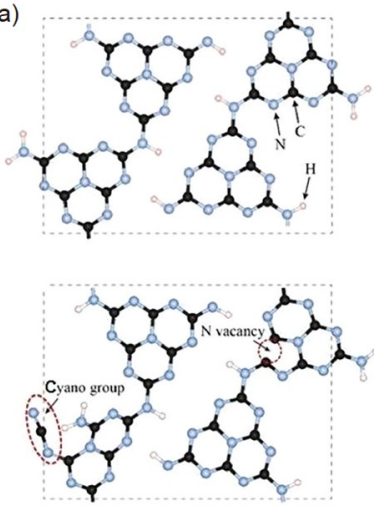
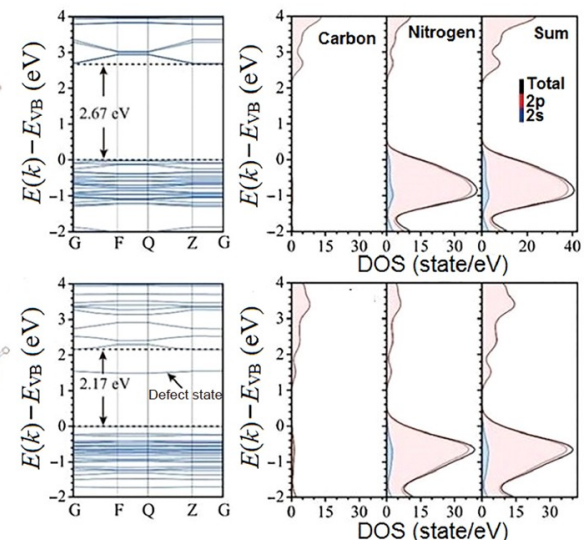

(b)

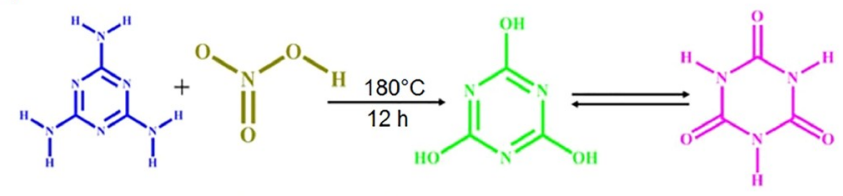

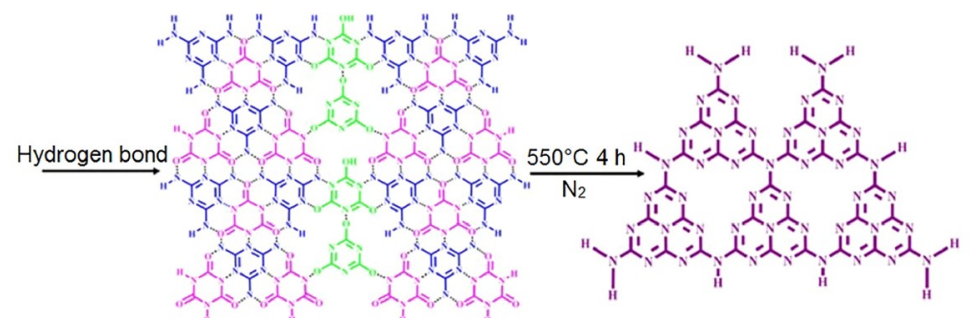

(c)

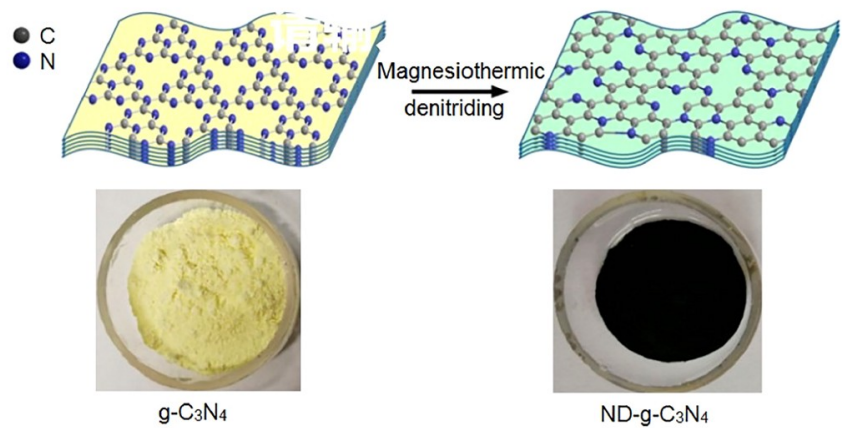

图 2 (网络版彩色)缺陷型石墨相氮化碳的制备机理图. (a) KOH辅助制备缺陷g- $\mathrm{C}_{3} \mathrm{~N}_{4}$ 机理、g- $\mathrm{C}_{3} \mathrm{~N}_{4}$ 与缺陷g- $\mathrm{C}_{3} \mathrm{~N}_{4}$ 的能带结构和DOS图谱 ${ }^{[84]}$; (b) 硝酸辅助制备缺陷 $g-C_{3} \mathrm{~N}_{4}$ 机理 ${ }^{[85]} ;$ (c) g- $\mathrm{C}_{3} \mathrm{~N}_{4}$ 的镁热脱氮合成ND-g- $\mathrm{C}_{3} \mathrm{~N}_{4}$ 的示意图以及镁热脱氮前的 $g-C_{3} \mathrm{~N}_{4}$ 和镁热脱氮后的 $N D-g-C_{3} N_{4}$ 照片 ${ }^{[93]}$ Figure 2 (Color online) Preparation mechanisms of defective graphite carbon nitride. (a) Mechanism of KOH-assisted preparation of nitrogencontaining defects $g-\mathrm{C}_{3} \mathrm{~N}_{4}$, energy band structures and DOS spectra of carbon nitride and defectiveg- $\mathrm{C}_{3} \mathrm{~N}_{4}{ }^{[84]}$; (b) mechanism of nitric acid-assisted preparation of defective $\mathrm{g}-\mathrm{C}_{3} \mathrm{~N}_{4}^{[85]}$; (c) schematic diagram of the synthesis of ND-g- $\mathrm{C}_{3} \mathrm{~N}_{4}$ by magnesium thermal denitrification of $\mathrm{g}-\mathrm{C}_{3} \mathrm{~N}_{4}$ and photos of g- $\mathrm{C}_{3} \mathrm{~N}_{4}$ before magnesium pyrolysis of nitrogen and ND-g- $\mathrm{C}_{3} \mathrm{~N}_{4}$ after magnesium pyrolysis of nitrogen ${ }^{[93]}$

块体在 $475 \sim 550^{\circ} \mathrm{C}$ 加热，制备了不同氮空位密度的 $\mathrm{g}-\mathrm{C}_{3} \mathrm{~N}_{4}$ 纳米片, 相应的样品标记为 $\mathrm{CN}-T$, 其中 $T$ 表示热 处理温度(CN-475、CN-500、CN-525和CN-550). 所获 得 $\mathrm{CN}-525$ 的 $\mathrm{CO}_{2}$ 还原速率最高, 为 $6.21 \mu \mathrm{mol} / \mathrm{h}$, 是原始 $\mathrm{g}-\mathrm{C}_{3} \mathrm{~N}_{4}(1.48 \mu \mathrm{mol} / \mathrm{h})$ 的 4.2 倍; CN-525在水分解过程中, 氢气的析出速率最高, 为 $64.39 \mu \mathrm{mol} / \mathrm{h}$, 是原始 $\mathrm{g}-\mathrm{C}_{3} \mathrm{~N}_{4}$ 的
18倍.

\section{3 聚合后调节}

熔盐作为液体反应介质，对含氧或其他含杂原子 ( $\mathrm{S}$ 和 $\mathrm{N}$ 等)的物质具有很强的溶解能力, 从而增强了这 些物质的迁移和反应能力, 熔盐也为反应提供了独特 
的惰性气氛. 一般来说, 熔盐可以作为石墨材料合成的 溶剂、剪裁微观和介孔材料的软模板，以及缩聚和脱 氨反应中的结构导向剂. Zhang等人 ${ }^{[90]}$ 将尿素和草酰胺 共缩合物在熔融盐中进行后炦烧, 得到具有超高光催 化制氢活性的 $\mathrm{g}-\mathrm{C}_{3} \mathrm{~N}_{4}$. 结果表明, 尿素和酰胺在熔融盐 中共缩合后, 在熔融盐中煅烧, 七嗪单元的 $\pi-\pi$ 层堆积距 离最大为 $0.292 \mathrm{~nm}$, 改善了横向电荷输运和层间激子离 解. 酰胺的加人使禁带宽度从 $2.74 \mathrm{eV}$ 减小到 $2.56 \mathrm{eV}$, 使 得材料在绿光下也能实现高效的光催化. 在 420 和 $525 \mathrm{~nm}$, 优化后样品的析氢表观量子产率分别达到 $57 \%$ 和 $10 \%$, 明显高于以往的实验结果.

$\mathrm{Liu}^{\text {等人 }}{ }^{[91]}$ 发现在缩聚和脱氨反应中加人熔盐对 提高 $g-C_{3} N_{4}$ 的聚合程度具有促进作用. 与块状 $g-C_{3} N_{4}$ 相 比, 采用熔盐处理方法成功制备了缺陷 $\mathrm{g}-\mathrm{C}_{3} \mathrm{~N}_{4}$. 将 $\mathrm{KCl}$ 与 $\mathrm{LiCl}$ 的摩尔比为 $41: 59$ 的共晶混合物用作反应的高温 溶剂, 熔点约为 $352^{\circ} \mathrm{C}$. 将 $\mathrm{g}-\mathrm{C}_{3} \mathrm{~N}_{4}(1 \mathrm{~g})$ 与 $\mathrm{KCl}(8.2 \mathrm{~g})$ 和 $\mathrm{LiCl}$ $(11.8 \mathrm{~g})$ 一起在玛瑙研钵中研磨, 然后将得到的混合物 在马弗炉中以 $2^{\circ} \mathrm{C} / \mathrm{min}$ 的速率升温至 $550^{\circ} \mathrm{C}$, 并保存 $2 \mathrm{~h}$. 产品自然冷却至室温后煮沸, 再用去离子水彻底清洗 以去除残留的盐分, 在 $60^{\circ} \mathrm{C}$ 下干燥 $12 \mathrm{~h}$ 制得缺陷 $\mathrm{g}-\mathrm{C}_{3} \mathrm{~N}_{4}$. 缺陷 $\mathrm{g}-\mathrm{C}_{3} \mathrm{~N}_{4}$ 样品显示出稳定的光催化析氢速 率, 高达 $403.1 \mu \mathrm{mol} / \mathrm{h}$, 约为块状 $\mathrm{g}-\mathrm{C}_{3} \mathrm{~N}_{4}$ 的 2.2 倍, 归因于 缺陷 $\mathrm{g}-\mathrm{C}_{3} \mathrm{~N}_{4}$ 的聚合度提高、带隙降低及载流子有效分 离的协同作用效果.

通过二次热处理法也可制备缺陷 $g-\mathrm{C}_{3} \mathrm{~N}_{4}$. Liang等 人 ${ }^{[92]}$ 采用简单的二次热处理工艺制备了缺陷 $g-\mathrm{C}_{3} \mathrm{~N}_{4}$. 将尿素加热至 $550^{\circ} \mathrm{C}$ 并保持 $2 \mathrm{~h}$, 得到黄色 $\mathrm{g}-\mathrm{C}_{3} \mathrm{~N}_{4}$ 样品, 命名为 $\mathrm{g}-\mathrm{C}_{3} \mathrm{~N}_{4}(550)$. 将黄色样品置于带盖的陶瓷柜中, 在 $\mathrm{N}_{2}$ 氛围下热处理 $2 \mathrm{~h}$, 得到的样品命名为 $g-\mathrm{C}_{3} \mathrm{~N}_{4}(550$ $650)$, 合成的 $g-\mathrm{C}_{3} \mathrm{~N}_{4}(550-650)$ 比表面积为 $84 \mathrm{~m}^{2} / \mathrm{g}$. 结果 表明, 经过两次热处理, 产物的比表面积增大, 并且由 于氮缺陷的引人，其可见光捕获能力和光生载流子的 分离效率都得到了提高. 这些有益因素改善了缺陷g$\mathrm{C}_{3} \mathrm{~N}_{4}$ 在可见光下光催化制氢和降解各种染料的能力. Chen等人 ${ }^{[93]}$ 将 $10 \mathrm{~g}$ 原始 $\mathrm{g}-\mathrm{C}_{3} \mathrm{~N}_{4}$ 和 $5 \mathrm{~g}$ 镁粉混合在一起, 并装人带有不锈钢盖的不锈钢坩埚中. 然后将混合物 在管式炉中在流动的 $\mathrm{Ar}$ 气氛下加热至 $750^{\circ} \mathrm{C}$, 并在此温 度下保存 $2 \mathrm{~h}$. 冷却后, 在室温下依次用稀乙酸和蒸馏水 洗涤残留的镁和生成的副产物, 通过真空干燥获得缺 陷g- $\mathrm{C}_{3} \mathrm{~N}_{4}\left(\mathrm{ND}-\mathrm{g}-\mathrm{C}_{3} \mathrm{~N}_{4}\right)$ (图2(c)).

$\mathrm{Ma}$ 等人 ${ }^{\left[{ }^{[4]}\right.}$ 以双氰胺为前驱体, 将制备好的原始g$\mathrm{C}_{3} \mathrm{~N}_{4}$ 在 $\mathrm{HCl}$ 溶液中搅拌溶解, 把得到的白色悬浮液通过
加热干燥洗涤, 获得了含氮缺陷的 $\mathrm{g}-\mathrm{C}_{3} \mathrm{~N}_{4}$ 催化剂. 这些 氮空位不仅是吸附和活化 $\mathrm{N}_{2}$ 分子的活性中心，而且能 够促进 $g-\mathrm{C}_{3} \mathrm{~N}_{4}$ 向 $\mathrm{N}_{2}$ 分子的界面电荷转移. 当 $\mathrm{N}_{2}$ 分子与 氮空位相互作用时，其吸附能为 $166.2 \mathrm{~kJ} / \mathrm{mol} ， \mathrm{~N} \equiv \mathrm{N}$ 键 从1.107 $\AA$ 延长至 $1.242 \AA$, 证实氮空位可以激活 $\mathrm{N}_{2}$ 分 子. 通过溶剂热-酸处理合成的缺陷 $g-\mathrm{C}_{3} \mathrm{~N}_{4}$ 的 $\mathrm{NH}_{4}{ }^{+}$生成 速率达到 $6.32 \mathrm{mg} /\left(\mathrm{L} \mathrm{h} \mathrm{g}_{\text {cat }}\right)$, 是原始 $\mathrm{g}-\mathrm{C}_{3} \mathrm{~N}_{4}$ 的 13.4 倍.

\section{3 缺陷型石墨相氮化碳的光催化机制}

通常情况下, 光催化可分为 4 个主要过程, 涉及 7 个 阶段, 如图3所示. 4 个主要过程分别为: (1) 光收集(1阶 段); (2) 电荷激发(2阶段); (3) 电荷分离和转移(3 5阶 段); (4) 表面催化反应(6、7阶段). 其中, 催化剂的表面 形态和结构对光收集过程有很大的影响, 为了更好地 提高催化剂的光收集能力, 在催化剂中构建分层的大 孔或中孔结构能够更好地利用光的多重反射和散射效 果 ${ }^{[95]}$. 当照射的光高于或等于带隙能时, 半导体价带中 的 $\mathrm{e}^{-}$会被激发到导带, 价带上产生 $\mathrm{h}^{+}$, 在半导体材料中 形成的电子空穴对是一个具有高活性的氧化还原体系. 电荷的分离和转移是光催化量子效率的决定性因素, 4、5阶段分别表示半导体主体和表面的电荷重组，电 荷重组不利于电荷分离和转移(第3阶段)到表面活性位

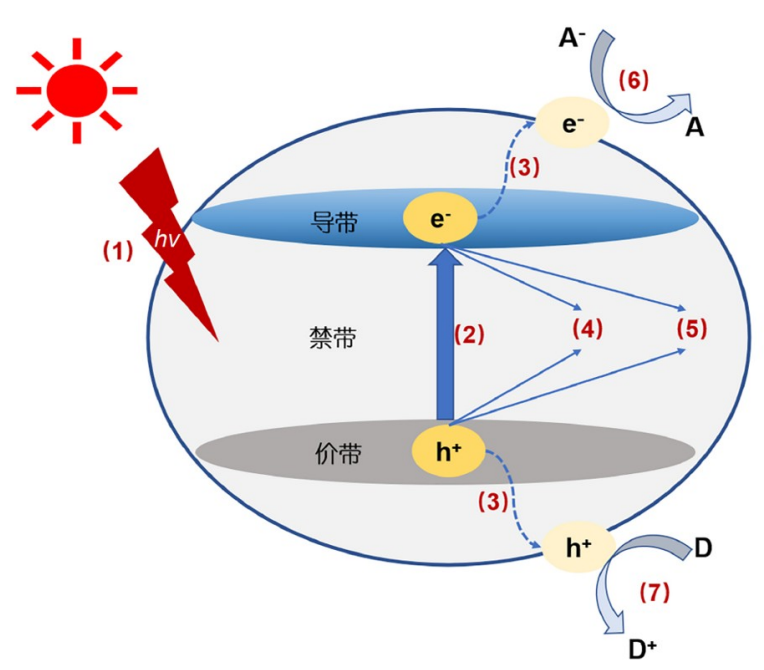

图 3 (网络版彩色)多相光催化的基本机理. 典型阶段: (1) 光收集阶 段; (2) 电荷激发; (3) 电荷分离和转移; (4) 批量电荷重组; (5) 表面电 荷复合; (6) 表面还原反应; (7) 表面氧化反应

Figure 3 (Color online) Basic mechanism of heterogeneous photocatalysis. Typical stages: (1) Light harvesting stage; (2) charge excitation; (3) charge separation and transfer; (4) bulk charge recombination; (5) surface charge recombination; (6) surface reduction reaction; (7) surface oxidation reaction 
点. 一般情况下, 构建界面电场或缩短光生载流子的扩 散长度可以使复合速率降低. 未发生重组的电子空穴 对可以进一步被助催化剂或表面活性部位捕获，从而 进行表面催化反应过程.

当光催化反应作用于处理对象时，半导体光催化 剂表面吸附的溶解氧能够在 $\mathrm{e}^{-}$的作用下, 生成 $\mathrm{O}_{2}{ }^{-}$, 材 料表面的 $\mathrm{OH}^{-}$和水分子捕获 $\mathrm{h}^{+}$从而被氧化成 $\mathrm{OH}$ ，具有 超强氧化性的. $\mathrm{O}_{2}{ }^{-}$和. $\mathrm{OH}$ 可以将处理对象氧化为 $\mathrm{CO}_{2}$ 和 $\mathrm{H}_{2} \mathrm{O}$ 等. 当处理对象为有机酸时, $\mathrm{h}^{+}$起作用, 发生氧化反 应. 当处理对象为高价态重金属离子时, $\mathrm{e}^{-}$起作用，发 生还原反应.

涉及的相关化学方程式如下:

$$
\begin{aligned}
& \text { 半导体光催化剂 }+h v \rightarrow \mathrm{e}^{-}+\mathrm{h}^{+} \\
& \mathrm{H}_{2} \mathrm{O}+\mathrm{h}^{+} \rightarrow \cdot \mathrm{OH}+\mathrm{H}^{+} \\
& \mathrm{OH}^{-}+\mathrm{h}^{+} \rightarrow \cdot \mathrm{OH}+\mathrm{H}^{+} \\
& \mathrm{O}_{2}+\mathrm{e}^{-} \rightarrow \cdot \mathrm{O}_{2}{ }^{-} \\
& \mathrm{O}_{2}{ }^{-}+2 \mathrm{H}^{+}+\mathrm{e}^{-} \rightarrow \mathrm{H}_{2} \mathrm{O}_{2} \\
& \mathrm{H}_{2} \mathrm{O}_{2}+\mathrm{e}^{-} \rightarrow \cdot \mathrm{OH}+\mathrm{OH}^{-} \\
& \mathrm{H}_{2} \mathrm{O}+\cdot \mathrm{O}_{2}^{-} \rightarrow \cdot \mathrm{OH}+\mathrm{OH}^{-}+\mathrm{O}_{2} \\
& \mathrm{Cr}^{6+}+3 \mathrm{e}^{-} \rightarrow \mathrm{Cr}{ }^{3+}
\end{aligned}
$$

然而，原始g- $\mathrm{C}_{3} \mathrm{~N}_{4}$ 表面的活性位点少，可见光吸收 范围有限, 并且光激发电荷载流子复合速率高, 从而导 致光催化活性较低. 光催化活性通常取决于光收集、 电荷传输和分离以及催化氧化过程的热力学和动力学 之间的平衡. $\mathrm{g}-\mathrm{C}_{3} \mathrm{~N}_{4}$ 在化学还原过程中产生了氮缺陷, 产生的氮缺陷分为氮空位缺陷 $\mathrm{N}_{2 \mathrm{C}}$ 和 $\mathrm{NH}_{x}$, 两种缺陷均 能产生局部能级. 氮空位缺陷产生的局部能级是深能 级, 其能够将光吸收范围扩展到可见光区; 氮空位缺陷 产生的局部能级是刚好高于价带最大值的浅能级, 能 够提高载流子迁移率并增加载流子扩散自由程 ${ }^{[96]}$. 氮 缺陷在缺陷 $\mathrm{g}-\mathrm{C}_{3} \mathrm{~N}_{4}$ 中产生了 3 个显著的特征：(1) 氮缺 陷诱导的中隙态可以转变为电子空穴激发的活性中心, 并由于带隙的变窄而拓宽了缺陷 $g-\mathrm{C}_{3} \mathrm{~N}_{4}$ 的光学响应范 围; (2) 氮缺陷可以捕获光生载流子并阻止其复合，从 而提高整体量子效率; (3) 由于带隙变窄(即导带(conduction band, CB)下移和价带(valence band, VB)上移), 缺陷 $\mathrm{g}-\mathrm{C}_{3} \mathrm{~N}_{4}$ 的氧化还原能力降低.

以 $\mathrm{NaBH}_{4}$ 和 $g-\mathrm{C}_{3} \mathrm{~N}_{4}$ 为前驱体通过固相化学还原技 术制备的缺陷 $\mathrm{g}-\mathrm{C}_{3} \mathrm{~N}_{4}$ 为例, 随着还原温度的升高, 缺陷
$\mathrm{g}-\mathrm{C}_{3} \mathrm{~N}_{4}$ 的颜色越来越深，当还原温度升高到 $500^{\circ} \mathrm{C}$ 时变 为褐色 ${ }^{[97]}$. 由图 $4(\mathrm{a})$ 可知, 随着还原温度的升高, $\mathrm{C} / \mathrm{N}$ 值 增加，氮缺陷明显增多. 图4(b), (c)显示了原始 $\mathrm{CN}$ 和 $\mathrm{CN}-400$ 的C 1s 和N 1s XPS光谱. C 1s XPS的3个分峰 分别位于 $288.2 、 286.2 、 284.7 \mathrm{eV}$ ，分别对应 $\mathrm{C}-\mathrm{N}=\mathrm{C}$ 、 $\mathrm{C}-\mathrm{NH}_{x}(x=1,2)$ 和 $\mathrm{C}-\mathrm{C} / \mathrm{C}=\mathrm{C} . \mathrm{CN}$ 的 $\mathrm{N} 1 \mathrm{~s}$ XPS谱显示了 3 个拟合峰，分别位于 $398.7 、 400.2$ 和 $401 \mathrm{eV}$ ，分别属于 $\mathrm{C}-\mathrm{N}-\mathrm{H}_{x} 、 \mathrm{~N}_{2 \mathrm{C}}(\mathrm{C}-\mathrm{N}=\mathrm{C})$ 和 $\mathrm{N}_{3 \mathrm{C}}\left(\mathrm{N}-(\mathrm{C})_{3}\right)$ 的特征峰. 图4(d) 展示了 $\mathrm{N}_{2 \mathrm{C}}$ 与 $\mathrm{N}_{3 \mathrm{C}}$ 的比值随还原温度的升高明显下降, 表明氮缺陷优先在 $\mathrm{N}_{2 \mathrm{C}}$ 位置产生. 与原始 $\mathrm{CN}$ 样品相比, 不同温度下制备的 $\mathrm{CN}-T$ 的吸收边缘有明显的红移. 此 外, 在400 900 nm范围内, 随着还原温度的升高, CN-T 的吸光能力逐渐增强. 实际上, 红移一般与氰基的存在 同步, 而氮空位会产生新的吸收边缘 ${ }^{[98]}$. 因此, $C N-T$ 的 红移和吸光能力的提升与氧基和氮空位有关. 经化学 还原处理后, BET比表面积从 CN的 $28.35 \mathrm{~m}^{2} / \mathrm{g}$ 逐渐增加 到CN-500的 $41.72 \mathrm{~m}^{2} / \mathrm{g}$, BET比表面积影响活性位点的 数量 ${ }^{[93]}$ 和界面电荷转移量子效率 ${ }^{[94]} . \mathrm{CN}-T$ 的 $\mathrm{H}_{2}$ 演化速 率远远大于 $\mathrm{CN}$, 可知比表面积对 $\mathrm{CN}-T$ 的光催化效果具 有一定影响.

光生电子空穴对的分离效率对提高光催化性能至 关重要, 通过引人一定量的氮缺陷, 对提升光生电子空 穴对的分离效率具有促进作用. 通过测量沉积在ITO电 极上样品的电化学阻抗谱和光电流响应, 可验证氮缺 陷对光致载流子分离和传输的影响. 在Wang等人 ${ }^{[85]}$ 的 研究中获得的缺陷 $g-\mathrm{C}_{3} \mathrm{~N}_{4}$ 的Rct值远小于普通 $g-\mathrm{C}_{3} \mathrm{~N}_{4}$, 表明缺陷的存在可有效增强 $\mathrm{g}-\mathrm{C}_{3} \mathrm{~N}_{4}$ 的电荷传递能力. 同时，该工作发现随着煅烧温度的增加，所制备缺陷 g- $\mathrm{C}_{3} \mathrm{~N}_{4}$ 的Rct值呈现先下降后上升的趋势，表明缺陷位 点的过量引人反而会抑制 $\mathrm{g}-\mathrm{C}_{3} \mathrm{~N}_{4}$ 的电荷传递效率. 通 过光电流分析，证实了氮缺陷对电子空穴对的复合有 抑制作用. 但过量引人氮缺陷会产生更深的中隙态从 而充当光生电荷复合中心. 这些较深的中隙态不仅可 以捕获光生电子, 还可以捕获光生 $\mathrm{h}^{+}$, 然后成为光生载 流子的重组位置. 在 $\mathrm{Tu}$ 等人 ${ }^{[89]}$ 的研究中, $\mathrm{CN}-525$ 电极 的光电流密度是所有样品中最大的, 光电流研究的结 果与 $\mathrm{CN}$ 和 $\mathrm{CN}-T$ 催化性能的变化趋势一致. 光致发光 (photoluminescence, PL) 光谱分析可以用来考察光生载 流子在 $\mathrm{CN}$ 或 $\mathrm{CN}-T$ 中的迁移、转移和分离效率, 因为半 导体的PL发射主要来自电荷-载流子的复合 ${ }^{[99]}$, PL值越 高，则半导体的电荷载流子越容易发生复合. 从原始 $\mathrm{CN}$ 到 CN-525, PL值逐步降低, 即光致载流子的复合强 

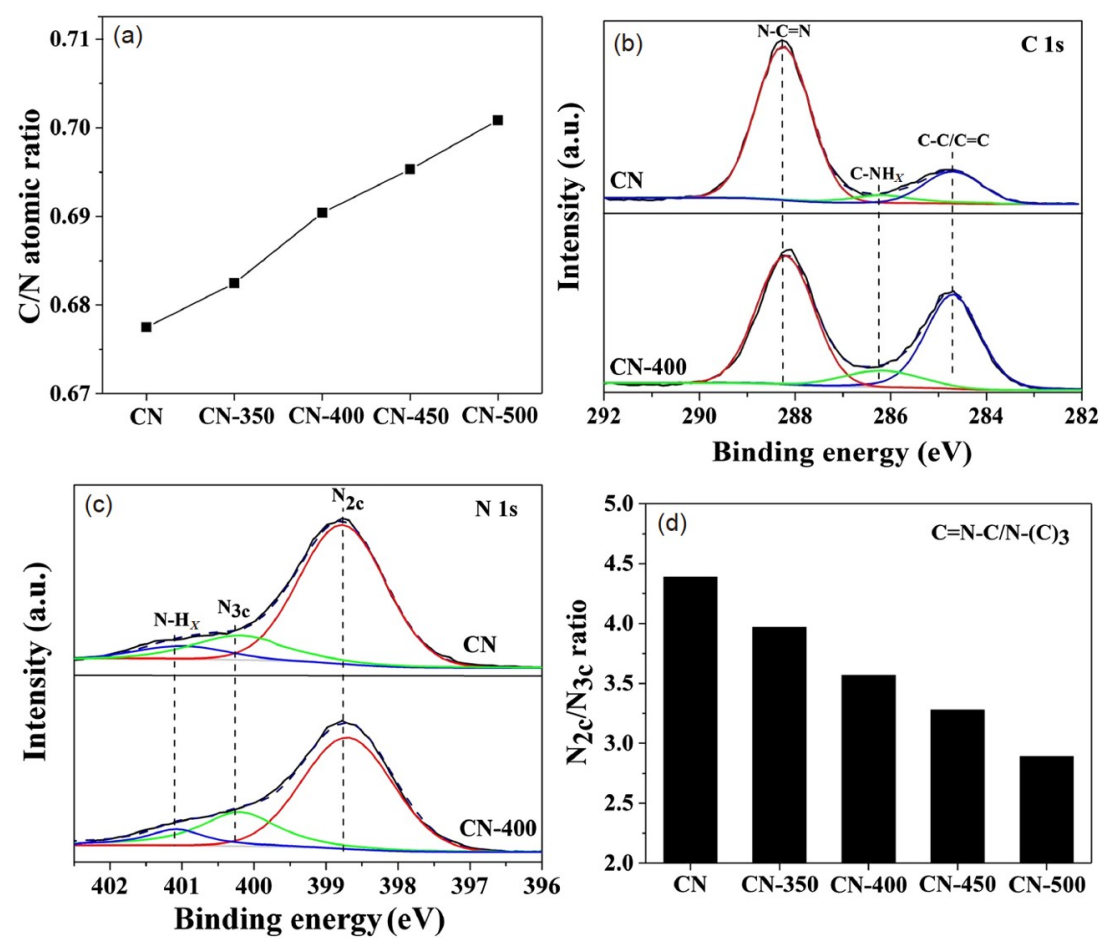

图 4 (网络版彩色) 原始 $\mathrm{CN}$ 和CN-T样品的碳氮比(a)、C $1 \mathrm{~s}(\mathrm{~b})$ 和 $N$ 1s XPS光谱(c)以及 $\mathrm{N}_{2 \mathrm{C}} / \mathrm{N}_{3 \mathrm{C}}$ 比例 $(\mathrm{d})^{[97]}$

Figure 4 (Color online) The $\mathrm{C} / \mathrm{N}$ ratios of the pristine $\mathrm{CN}$ and $\mathrm{CN}-\mathrm{T}$ samples (a), C 1s (b) and N 1s (c) XPS spectra of pristine $\mathrm{CN}$ and $\mathrm{CN}-400$, and $\mathrm{N}_{2} / \mathrm{N}_{3 \mathrm{C}}$ ratios of the pristine $\mathrm{CN}$ and $\mathrm{CN}-T$ samples $(\mathrm{d})^{[97]}$

度随着氮缺陷的增加而降低, 但是氮缺陷的进一步增 加导致 PL 强度的增加( CN-550). 在所有样品中, $\mathrm{CN}-525$ 的发射强度最低, 因此, CN-525对电子空穴对 复合的抑制作用最好，使得其在所制得的光催化剂中 活性最高.

\section{4 缺陷型石墨相氮化碳的应用}

\section{1 在水处理中的作用}

工农业的迅速发展对水环境造成了许多污染，其 中, 一些具有蓄积性、残留性的有机污染物, 如除草 剂、杀虫剂等, 长期存在于水环境中. 这些物质用目前 常规的处理方法, 如消毒、过滤等, 达不到良好的处理 效果. 这类有机污染物在饮用水中越来越容易被检测 到, 且负荷和种类都在增加, 对人类健康和自然环境有 着潜在的危害. 光催化技术因其能够应用于处理水环 境中的污染物得到了广泛的关注. 光催化原理是基于 光催化剂在光照条件下具有的氧化还原能力从而净化 污染物, 具有氮缺陷的 $g-\mathrm{C}_{3} \mathrm{~N}_{4}$ 因其优越特性, 可被用于 处理水中污染物质, 如有机染料、抗生素、有机农药 及重金属等.
有机染料作为轻工业的必需品被大量使用, 对环 境造成一定的负面影响, 缺陷 $g-\mathrm{C}_{3} \mathrm{~N}_{4}$ 对有机染料的降 解具有促进作用. Liang等人 ${ }^{[92]}$ 将 $g-C_{3} N_{4}$ 和缺陷 $g-C_{3} N_{4}$ 对相同浓度的有机染料进行降解来对比二者的光催化 降解性能, 在相同的反应条件下, 缺陷 $\mathrm{g}_{-} \mathrm{C}_{3} \mathrm{~N}_{4}$ 降解亚甲 基蓝( $\mathrm{MB})$ 、罗丹明 $\mathrm{B}(\mathrm{RhB})$ 和甲基橙 $(\mathrm{MO})$ 的速率更快, 并且可以将有机染料降解得更加完全.

目前环境中抗生素污染状况日益严重, $\mathrm{g}-\mathrm{C}_{3} \mathrm{~N}_{4}$ 作 为一种高效绿色的催化剂, 可用于水体中四环素的去 除. Shi等人 ${ }^{[100]}$ 的研究表明，以三聚氰胺为前驱体，在 $\mathrm{KOH}$ 溶液中进行聚合, 生成的缺陷 $\mathrm{g}-\mathrm{C}_{3} \mathrm{~N}_{4}$ 样品的比表 面积为 $11.027 \mathrm{~m}^{2} / \mathrm{g}$, 高于原始 $\mathrm{g}-\mathrm{C}_{3} \mathrm{~N}_{4}\left(9.102 \mathrm{~m}^{2} / \mathrm{g}\right)$. 在 $\lambda>420 \mathrm{~nm}$ 的可见光照射下, 缺陷 $\mathrm{g}-\mathrm{C}_{3} \mathrm{~N}_{4}$ 样品对浓度为 $10 \mathrm{mg} / \mathrm{L}$ 的四环素溶液的降解率达 $57 \%$, 降解速率常数 为原始 $\mathrm{g}-\mathrm{C}_{3} \mathrm{~N}_{4}$ 的 1.58 倍. 图5 展示了光催化降解四环素 的机理. 但同时有研究显示, 过量的氮缺陷会产生更深 的中隙态, 从而充当光生载流子的复合中心, 抑制光催 化活性 $^{[101]}$.

由于有机农药具有蓄积性、长期性和难降解性等 特点, 作为非金属半导体新型光催化材料, $\mathrm{g}-\mathrm{C}_{3} \mathrm{~N}_{4}$ 因其 优良的特性可应用于有机农药的降解. Ma课题组 ${ }^{[102]}$ 将 


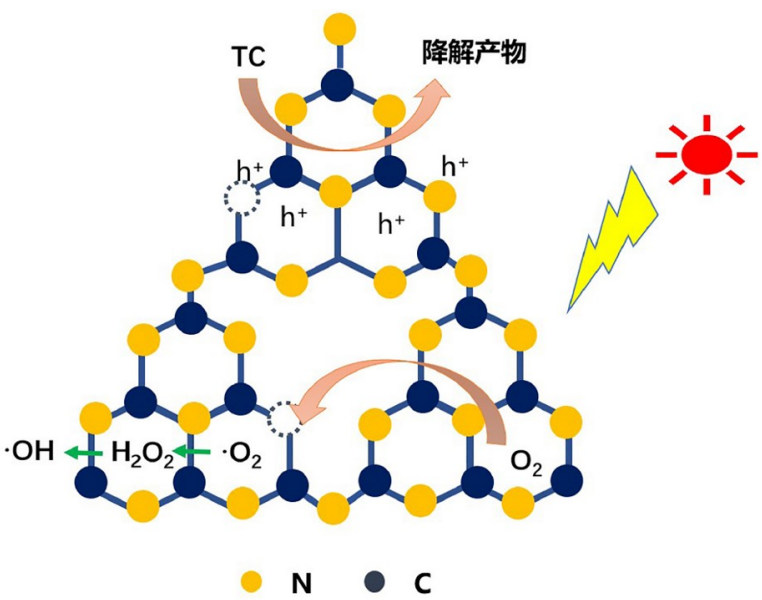

图 5 (网络版彩色)降解四环素的机理

Figure 5 (Color online) Degradation mechanism of tetracycline

改性后的 $\mathrm{g}-\mathrm{C}_{3} \mathrm{~N}_{4}$ 替代 $\mathrm{TiO}_{2}$ 来降解新烟碱类杀虫剂吡虫 啉, 这种物质对蜜蜂具有显著危害. 结果表明, 吡虫啉 在可见光条件下得到了快速降解. 为了测定不同果汁 样品中苯甲酰脲类农药, Guan等人 ${ }^{[103]}$ 将改性后的 $\mathrm{g}^{-} \mathrm{C}_{3} \mathrm{~N}_{4}$ 作为固相萃取吸附剂. 在最佳实验条件下, 苯甲 酰脲类农药的检测限为 $6 \mu \mathrm{g} / \mathrm{L}$, 回收率为 $70.4 \% \sim 96.4 \%$. 除此之外， $\mathrm{g}-\mathrm{C}_{3} \mathrm{~N}_{4}$ 纳米管显示出 10 倍的重复利用潜力. 结果表明, $\mathrm{g}-\mathrm{C}_{3} \mathrm{~N}_{4}$ 纳米管可用于污染物的富集和痕量 分析, 是一种有潜力的固相萃取吸附剂.

g- $\mathrm{C}_{3} \mathrm{~N}_{4}$ 还可以用于还原重金属, Wei等人 ${ }^{[104]}$ 研究 了以 $-\mathrm{C}_{3} \mathrm{~N}_{4}$ 作为催化剂对六价铬的降解效果, 在可见 光照射下, 光生电子可以将六价铬还原为三价铬, 其毒 性降低了百倍. 利用改性后的具有优良性能的缺陷 g- $\mathrm{C}_{3} \mathrm{~N}_{4}$ 处理水中的重金属是一种有效的方法. Zhang等 人 $^{[105]}$ 将制得的 $\mathrm{g}-\mathrm{C}_{3} \mathrm{~N}_{4}$ 在 $\mathrm{HCl}$ 或 $\mathrm{HNO}_{3}$ 中浸泡获得了酸 处理后的样品. 用 $5 \mathrm{~mol} / \mathrm{L} \mathrm{HNO}_{3}$ 和 $\mathrm{HCl}$ 处理的产物分别 表示为 $g-\mathrm{C}_{3} \mathrm{~N}_{4}-\mathrm{HNO}_{3}$ 和 $\mathrm{g}-\mathrm{C}_{3} \mathrm{~N}_{4}-\mathrm{HCl}$. 在柠檬酸存在的条 件下，光催化还原六价铬的能力从小到大依次为 g- $\mathrm{C}_{3} \mathrm{~N}_{4}<\mathrm{g}-\mathrm{C}_{3} \mathrm{~N}_{4}-\mathrm{HNO}_{3}<\mathrm{g}-\mathrm{C}_{3} \mathrm{~N}_{4}-\mathrm{HCl}$. 经过酸处理后, 获得的样品具有更大的比表面积，同时其表面正电荷 较大, 对 $\mathrm{Cr}(\mathrm{VI})$ 的吸附容量较大, 其光催化活性提高. $\mathrm{HNO}_{3}$ 处理后的 $\mathrm{g}-\mathrm{C}_{3} \mathrm{~N}_{4}$ 不仅可以有效地还原水溶液中 的 $\mathrm{Cr}(\mathrm{VI}), \mathrm{g}-\mathrm{C}_{3} \mathrm{~N}_{4}$ 的光催化再利用效率也得到提高.

\section{2 对水分解的促进作用}

氢能作为可再生的清洁能源，化石燃料制氢、电 解水制氢等是传统的制氢方法, 但很多时候, 这些方法 的原料利用率不高且需要消耗大量的能量. 光催化技
术是缓解这种现状的有效途径之一，光催化分解水制 氢能够节省常规能源. 缺陷 $\mathrm{g}-\mathrm{C}_{3} \mathrm{~N}_{4}$ 的比表面积更大, 可 为光催化反应提供的活性位点更多, 电荷载流子的迁 移能力更强, 具备良好的光催化性能. 缺陷 $\mathrm{g}-\mathrm{C}_{3} \mathrm{~N}_{4}$ 与其 他光催化剂制取氢气的生成速率 ${ }^{[106-110]}$ 如表S2所示.

Huang等人 ${ }^{[111]}$ 揭示了以Pt为助催化剂的缺陷 $\mathrm{g}$ $\mathrm{C}_{3} \mathrm{~N}_{4}$ 纳米片催化水解释放 $\mathrm{H}_{2}$ 的机理. 如图6所示, 氮空 位在导带边缘以下产生中空态，并且随着空位密度的 增加, 中空态的位置变深. 在具有一定能量的光照下, $\mathrm{g}-\mathrm{C}_{3} \mathrm{~N}_{4}$ 中处于价态上的电子不仅可以被激发迁移至导 带，而且还可以通过吸收更长波长的可见光而变为中 间能隙状态. 随着深能隙状态的形成, 吸收光的波长逐 渐变长, 这会激发更多的电子空穴对. 同时, 导带中被 激发的电子可以被中间能隙状态捕获，这对电子空穴 对的分离具有积极作用. 但是氮空位过量引人会引起 更深的中间能隙状态，电子空穴对的复合会发生在这 些中间隙态上. 最终, 被成功激发的电子转移到 Pt纳米 粒子上, 并将水还原为氢气.

\section{3 对二氧化碳的转化作用}

从发展可持续能源的角度来看，利用太阳能将温 室气体 $\mathrm{CO}_{2}$ 转化为有价值的能源物质(如 $\mathrm{CO}$ 、甲烷和 甲醇)将是解决全球变暖和化石燃料短缺两大问题的 最佳途径之一. 光催化技术可高效还原 $\mathrm{CO}_{2}$ 为 $\mathrm{CO}$ 、 $\mathrm{CH}_{4} 、 \mathrm{CH}_{3} \mathrm{OH} 、 \mathrm{HCHO}$ 和 $\mathrm{HCOOH}$ 等. 二氧化碳还原的 电位为

$$
\begin{aligned}
& \mathrm{CO}_{2}+2 \mathrm{H}^{+}+2 \mathrm{e}^{-} \rightarrow \mathrm{CO}+\mathrm{H}_{2} \mathrm{O} \quad(0.53 \mathrm{eV}) \\
& \mathrm{CO}_{2}+8 \mathrm{H}^{+}+8 \mathrm{e}^{-} \rightarrow \mathrm{CH}_{4}+2 \mathrm{H}_{2} \mathrm{O} \quad(0.24 \mathrm{eV}) \\
& \mathrm{CO}_{2}+6 \mathrm{H}^{+}+6 \mathrm{e}^{-} \rightarrow \mathrm{CH}_{3} \mathrm{OH}+\mathrm{H}_{2} \mathrm{O} \quad(0.38 \mathrm{eV}) \\
& \mathrm{CO}_{2}+4 \mathrm{H}^{+}+4 \mathrm{e}^{-} \rightarrow \mathrm{HCHO}+\mathrm{H}_{2} \mathrm{O} \quad(0.48 \mathrm{eV}) \\
& \mathrm{CO}_{2}+2 \mathrm{H}^{+}+2 \mathrm{e}^{-} \rightarrow \mathrm{HCOOH} \quad(0.61 \mathrm{eV})
\end{aligned}
$$

相比于原始 $\mathrm{g}-\mathrm{C}_{3} \mathrm{~N}_{4}$, 含氮空位缺陷 $\mathrm{g}-\mathrm{C}_{3} \mathrm{~N}_{4}$ 的催化性 能更加优异, 氮空位的掺杂作用可增强 $\mathrm{CO}_{2}$ 的光还原性 能，空穴诱导的中空穴态的固有特性有利于在两步过 程中介导多电子激发. 这有利于光吸收范围扩展到近 红外区域, 实现太阳能的最优利用.

为了测定 $\mathrm{CO}_{2}$ 在缺陷 $\mathrm{g}-\mathrm{C}_{3} \mathrm{~N}_{4}$ 催化剂上还原为 $\mathrm{CH}_{4}$ 的 还原效率, Tang等人 ${ }^{[112]}$ 设计了相关实验. 该反应为避 免外界光干扰在封闭黑暗环境中进行, 以 $500 \mathrm{~W}$ 氙弧灯 (CHF-XM-500 W) 为光源, 配以紫外截止滤光片 $(\lambda>$ 


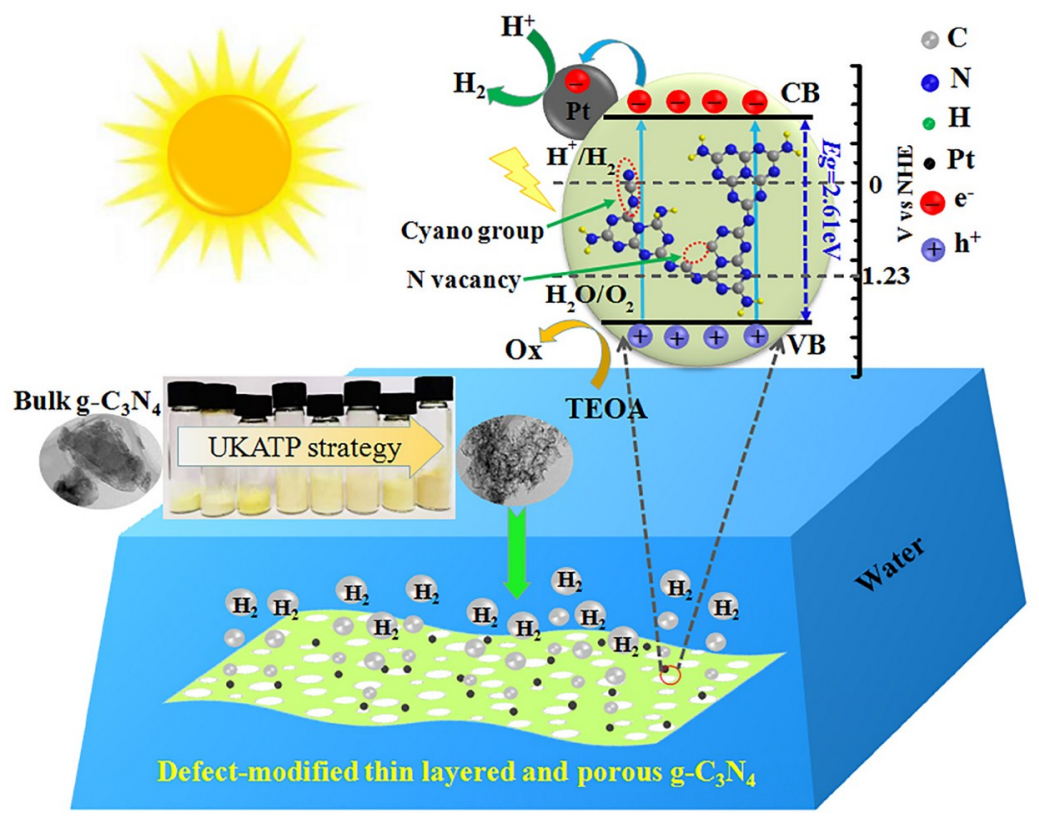

图 6 (网络版彩色)缺陷修饰的薄层多孔g- $\mathrm{C}_{3} \mathrm{~N}_{4}$ 光催化 $\mathrm{H}_{2}$ 演化机理研究 ${ }^{[111]}$

Figure 6 (Color online) Study on the mechanism of photocatalytic $\mathrm{H}_{2}$ evolution of defect modified thin-layer porous g- $\mathrm{C}_{3} \mathrm{~N}_{4}{ }^{[111]}$

$400 \mathrm{~nm}$ )模拟可见光辐射. 首先, 将缺陷 $g-\mathrm{C}_{3} \mathrm{~N}_{4}$ 样品光催 化剂的薄膜均匀地涂在玻璃棒上，将其装人石英柱夹 持器, 并直接置于光源下方. 以 $50 \mathrm{~mL} / \mathrm{min}$ 的流速用增 湿的 $\mathrm{CO}_{2}$ 吹扫系统, 以去除多余的空气, 并在光催化剂 表面达到 $\mathrm{CO}_{2}$ 吸附-解吸平衡. 此后, 增湿 $\mathrm{CO}_{2}$ 的流速降 低至 $5 \mathrm{~mL} / \mathrm{min}$, 维持 $8 \mathrm{~h}$. 利用气相色谱仪结合热导检测 器和火焰离子化检测器对光催化 $\mathrm{CO}_{2}$ 释放产生的气体 产物进行检测. 结果表明, 将氮缺陷引人 $\mathrm{g}-\mathrm{C}_{3} \mathrm{~N}_{4}$ 中时, $\mathrm{CH}_{4}$ 的生成速率提升了 5.14倍. 他们进一步揭示了 $\mathrm{CO}_{2}$ 在缺陷 $\mathrm{g}-\mathrm{C}_{3} \mathrm{~N}_{4}$ 催化剂上还原为 $\mathrm{CH}_{4}$ 的机理, 氮空位在增 强缺陷 $\mathrm{g}-\mathrm{C}_{3} \mathrm{~N}_{4}$ 催化剂光催化还原 $\mathrm{CO}_{2}$ 起到了关键作用. 如图7所示, 氮空位的存在导致了位于 $\mathrm{CB}$ 边缘附近的中 隙态, 带尾能量在1.17 1.32 eV之间. 这使得缺陷 $\mathrm{g}-\mathrm{C}_{3} \mathrm{~N}_{4}$ 能够在可见光区域捕获短波和长波光子, 并产生两步 电子激发过程. 短波光子在可见光区域占优势, 基态电 子主要被激发到 $\mathrm{CB}$ (路径1). 少数长波光子随后被吸收, 以直接激发电子至中隙态(路径2). 不可避免地, 一些处 于激发态的电子可能会失去能量并返回基态. 空位诱 导的中隙态将作为电子库来捕获这些不稳定的电子, 如路径3所示. 与自由激子相比, 位于中隙态电子的寿 命更长. 这反过来又会有效地阻碍电子空穴对在基态 的直接复合. 最终, 当提供足够的激发能时, 被俘获的 电子从中隙态激发到 $\mathrm{CB}$, 随后参与 $\mathrm{CO}_{2}$ 释放过程(路径
4). 然而, 考虑到中隙态的过度加深将促进俘获电子和 光生空穴的复合(路径5), 从而延缓二氧化碳的释放过 程, 氮空位的程度应该优化.

除此之外，二氧化碳也可通过水蒸气在 $\mathrm{g}-\mathrm{C}_{3} \mathrm{~N}_{4}$ 和 缺陷 $\mathrm{g}-\mathrm{C}_{3} \mathrm{~N}_{4}$ 催化剂上还原为 $\mathrm{CO} . \mathrm{Tu}$ 等人 ${ }^{[89]}$ 在氢气气氛 加热条件下制备了不同氮空位密度的 $\mathrm{g}-\mathrm{C}_{3} \mathrm{~N}_{4}$ 纳米片, 相应的样品标记为 $\mathrm{CN}-T$, 未经改性的 $\mathrm{g}-\mathrm{C}_{3} \mathrm{~N}_{4}$ 记为 $\mathrm{BCN}$. $\mathrm{CN}-525$ 还原CO的速率最高, 为 $6.21 \mu \mathrm{mol} / \mathrm{h}$, 是 $\mathrm{BCN}$ $(1.48 \mu \mathrm{mol} / \mathrm{h})$ 的 4.2 倍. 所有样品在3440 3530 G的磁场 中均显示一条 $g$ 值为 2.0021 的洛伦兹线, 这与 $\pi$ 共轭芳环 中 $\mathrm{sp}^{2}$-碳原子的未配对电子相关 ${ }^{[113]}$. 类似于 $\mathrm{TiO}_{2}$ 表面上 氧空位的作用, 它们会向 $\mathrm{Ti}$ 原子提供过量的未配对电 子, 并随后将 $\mathrm{Ti}^{4+}$ 还原为 $\mathrm{Ti}^{3+}$ 顺磁性物质. 两个双配位氮 原子空位的形成也可向 $\mathrm{g}-\mathrm{C}_{3} \mathrm{~N}_{4}$ 的 $\pi$ 共轭芳环内的相邻 $\mathrm{sp}^{2}$-碳原子提供未配对的电子. 与具有几乎可以忽略不 计的电子顺磁共振(electron paramagnetic resonance, EPR)信号的BCN相比, 缺陷 $\mathrm{g}-\mathrm{C}_{3} \mathrm{~N}_{4}$ 的EPR信号强度逐 渐增强, 表明在 $\mathrm{g}-\mathrm{C}_{3} \mathrm{~N}_{4}$ 中产生的氮空位增加. 大量的孤 对电子被困在氮空位中, 有利于 $\mathrm{CO}_{2}$ 活化生成 $\mathrm{CO}_{2}{ }^{-}$中 间产物, 促进 $\mathrm{CO}_{2}$ 光还原成 $\mathrm{CO}$. 然而, 氮空位的过度增 加导致CO的析出速率 $(4.5 \mu \mathrm{mol} / \mathrm{h})$ 略有下降. 结果表明, 最佳浓度范围内的氮空位与光催化活性呈正相关. 在 $\lambda>420 \mathrm{~nm}$ 、反应介质为 $\mathrm{CO}_{2}$ 和水蒸气条件下, 将优化的 


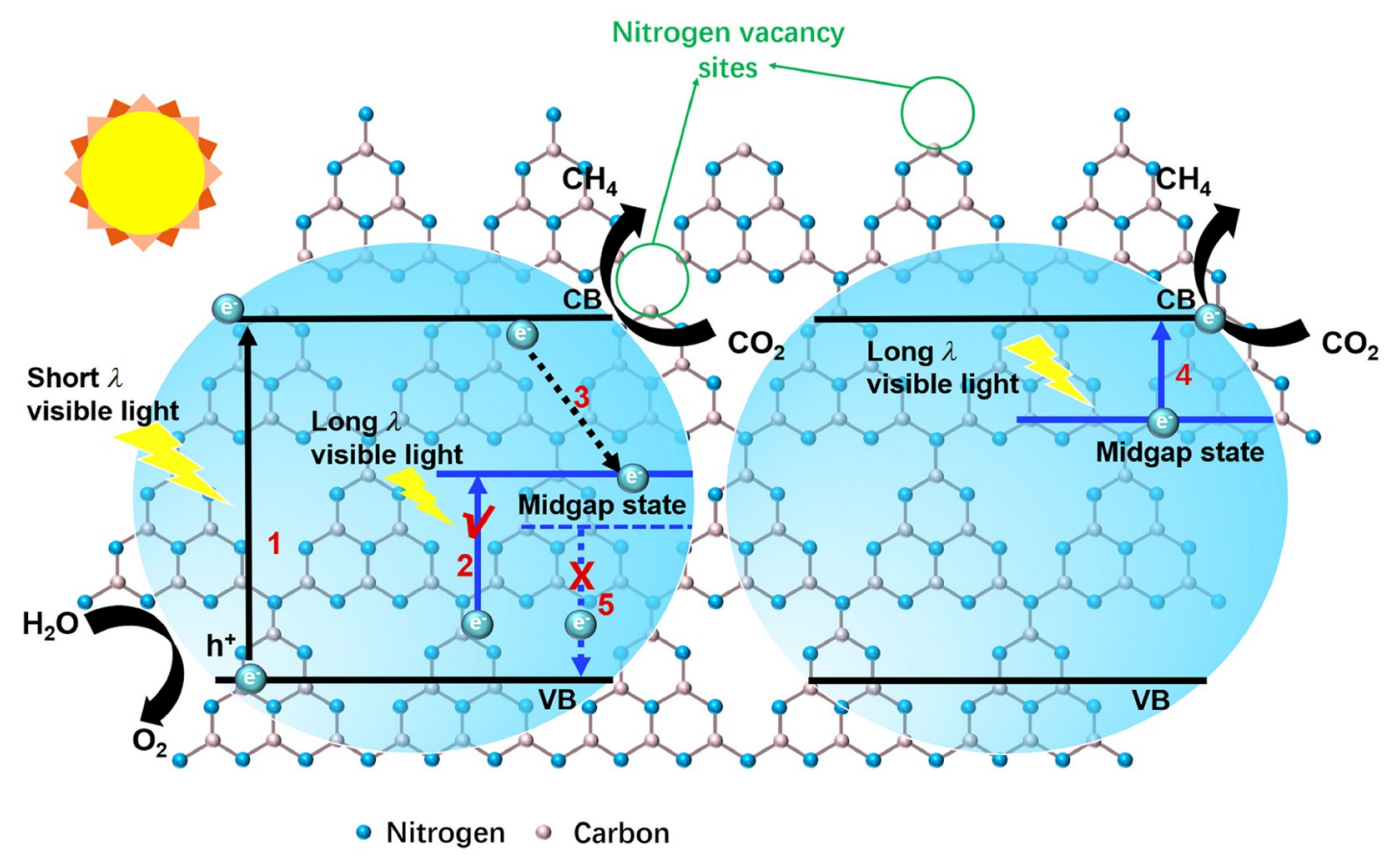

图 7 (网络版彩色) $\mathrm{CO}_{2}$ 在缺陷g- $\mathrm{C}_{3} \mathrm{~N}_{4}$ 催化剂上还原为 $\mathrm{CH}_{4}$ 的机理图 ${ }^{[112]}$

Figure 7 (Color online) The mechanism of $\mathrm{CO}_{2}$ reduction to $\mathrm{CH}_{4}$ on defective g- $\mathrm{C}_{3} \mathrm{~N}_{4}$ catalysts ${ }^{[112]}$

CN-525样品与其他氮化碳催化剂的催化活性进行比

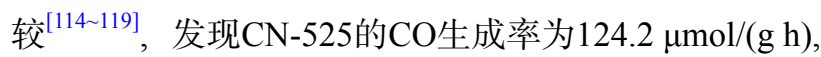
远高于其他氮化碳催化剂, 如表S3所示.

\section{4 在光催化脱氮中的作用}

开发能够有效活化目标污染物并促进其完全转化 的催化剂是环境光催化领域的一个目标, 研究 $\mathrm{g}-\mathrm{C}_{3} \mathrm{~N}_{4}$ 微管中氮空位对 $\mathrm{O}_{2}$ 和 $\mathrm{NO}$ 吸附活化、电子俘获和电子 结构的影响, 可以更好地利用光催化剂. Wang等人 ${ }^{[120]}$ 制备了氮空位浓度可调的 $\mathrm{g}-\mathrm{C}_{3} \mathrm{~N}_{4}$ 微管, 借助水热实验 得到CNT- $X$ ( $X$ 代表加热的时间，CNT-12代表加热 $12 \mathrm{~h}$ ), 详细讨论了 $\mathrm{g}-\mathrm{C}_{3} \mathrm{~N}_{4}$ 的形态演化, 即从块状结构到多孔 管状结构. 结果表明, 多孔管状结构 $g-\mathrm{C}_{3} \mathrm{~N}_{4}$ 微管的 $\mathrm{NO}$ 去除率和表观反应速率常数分别是原 $\mathrm{g}-\mathrm{C}_{3} \mathrm{~N}_{4}$ 的 1.8 和 2.6 倍.

根据ESR结果, $\mathrm{O}_{2}{ }^{-}$是参与 $\mathrm{NO}$ 去除的关键活性物 质. $\mathrm{O}_{2}{ }^{-}$的形成包括两个步骤: 在黑暗中材料对 $\mathrm{O}_{2}$ 的吸 附和在光照下吸附的 $\mathrm{O}_{2}$ 被光电子还原. 为了研究 $\mathrm{g}-\mathrm{C}_{3} \mathrm{~N}_{4}$ 和缺陷 $\mathrm{g}-\mathrm{C}_{3} \mathrm{~N}_{4}$ 对 $\mathrm{O}_{2}$ 吸附行为的差异, 采用密度 泛函理论(density functional theory, DFT)计算 $\mathrm{O}_{2}$ 和NO 的吸附能. 从热力学的角度来看, 分子 $\mathrm{O}_{2}$ 很难吸附到原 始 $\mathrm{g}-\mathrm{C}_{3} \mathrm{~N}_{4}\left(\Delta E_{\mathrm{ads}}=0.48 \mathrm{eV}\right)$, 相比之下, $\mathrm{O}_{2}$ 可以自发地吸 附在缺陷 $\mathrm{g}-\mathrm{C}_{3} \mathrm{~N}_{4}\left(\Delta E_{\mathrm{ads}}=-5.99 \mathrm{eV}\right)$. $\mathrm{NO}$ 分子很难吸附到
原始 $\mathrm{g}-\mathrm{C}_{3} \mathrm{~N}_{4}\left(\Delta E_{\mathrm{ads}}=0.29 \mathrm{eV}\right)$, 但是在缺陷 $\mathrm{g}-\mathrm{C}_{3} \mathrm{~N}_{4}$ 上可以 自发地被吸附 $\left(\Delta E_{\mathrm{ads}}=-5.91 \mathrm{eV}\right)$. 除此之外, $\mathrm{O}_{2}$ 分子最 终在氮空位处分解为两个活性的 $\mathrm{O}$ 原子, 这些活跃的 $\mathrm{O}$ 原子更容易被光生电子还原为 $\cdot \mathrm{O}_{2}{ }^{-}$. 这些结果表明，在 后续的光反应过程中, 氮空位不仅增加了 $\mathrm{O}_{2}$ 和NO吸附 能力, 而且实现了 $\mathrm{O}_{2}$ 分子的解离活化, 降低了 $\cdot \mathrm{O}_{2}{ }^{-}$形成 的障碍.

为了说明在管状结构中引人氮空位后光电分离效 率的变化, 记录了原始 $\mathrm{CN}$ 和 CNT样品的瞬态光电流和 PL光谱. 由图8(a)可知, 碳纳米管样品所产生的光电流 普遍高于块状 $\mathrm{CN}$, 说明碳纳米管样品具有较薄的空 管结构, 具有较好的光致电荷分离能力. 光电流从 CNT-8的平均值 $0.59 \mu \mathrm{A} / \mathrm{cm}^{2}$ 增加到 CNT-12的平均值 $1.12 \mu \mathrm{A} / \mathrm{cm}^{2}$, 这是因为后者的BET表面积更大, 从而提 供了更多的表面氮空位. 由于CNT-16中有序七嗪环骨 架结构的过度损失及其大量高浓度氮缺陷, CNT-16的 光电流随后降至 $0.90 \mu \mathrm{A} / \mathrm{cm}^{2}$. 为了进一步了解不规则 块体形态与管状结构对电荷运输能力的影响, 分析了 样品的电化学阻抗谱, CNT样品的Nyquist曲线半径比 CN小, 如图8(d)所示, 结果说明一维管状结构有利于载 流子的纵向运输. 在管状结构中, CNT-12弧半径最小, 由于其具有合适的薄壁结构和纵向结构，其电荷转移 效率最高. 上述结果表明, 适量的表面氮空位和一维管 
状结构能够对电子空穴对的分离有促进作用，从而提 高光催化活性.

$\mathrm{NO}$ 在原始和缺陷 $g-\mathrm{C}_{3} \mathrm{~N}_{4}$ 上的反应途径和反应位点 不同, 如图9所示, $\mathrm{NO}$ 和 $\mathrm{O}_{2}$ 在 $\mathrm{N}-\mathrm{H}$ 键处吸收, 在原始g$\mathrm{C}_{3} \mathrm{~N}_{4}$ 上形成 $\mathrm{NOH}$ 和 $\mathrm{OOH}$ 键(图9, 左图(I)). 然后在吸附 过程中被活化形成 $\mathrm{NO}^{-}$和表面过氧物质(图9, 左图(II)).

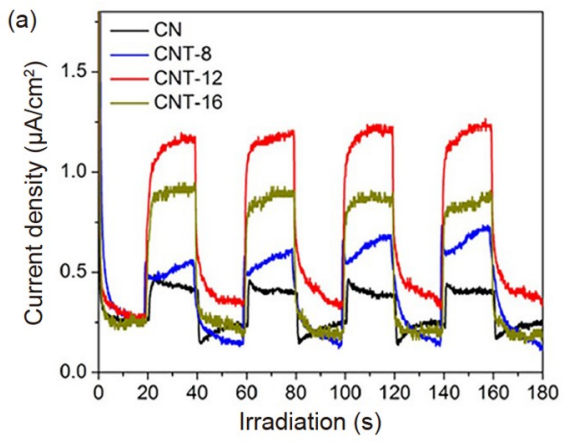

(c)

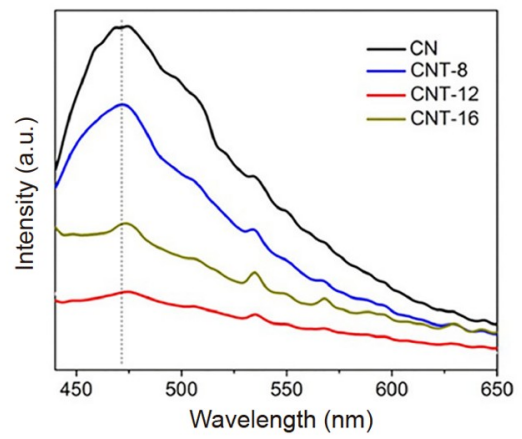

在光照下，过氧物质被光生电子还原生成· $\mathrm{O}_{2}{ }^{-}$(图9, 左 图(III)), 吸收态的中间体在 $\mathrm{N}$ 空位被 $\cdot \mathrm{O}_{2}{ }^{-}$和 $\mathrm{h}^{+}$的作用下 氧化为 $\mathrm{NO}_{3}{ }^{-}$(图9, 左图(IV)). 然而, 缺陷 $\mathrm{g}-\mathrm{C}_{3} \mathrm{~N}_{4}$ 上的 $\mathrm{NO} 、 \mathrm{O}_{2}$ 吸附和光催化通过不同的途径进行. $\mathrm{NO}$ 和 $\mathrm{O}_{2}$ 在N空位处通过双配位基可以吸收更快(图9, 右图(I)), 而不是在末端 $\mathrm{N}-\mathrm{H}$ 键处吸收. 这些物质被激活并形成

(b)

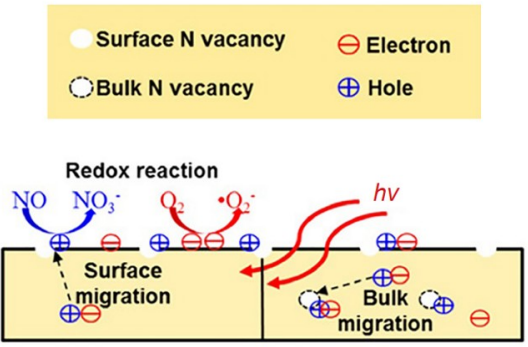

(d)

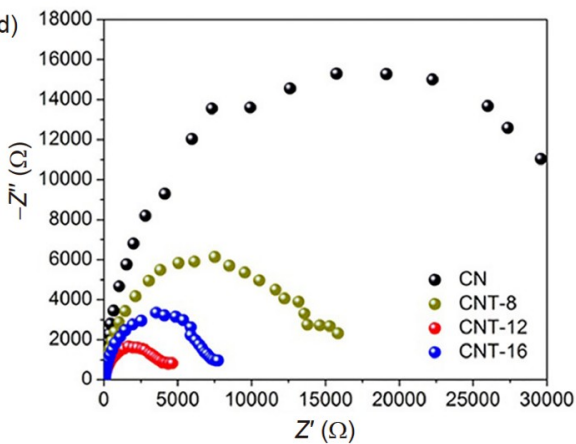

图 8 (网络版彩色)CN、CNT-8、CNT-12、CNT-16的瞬态光电流响应(a), 描述N空位在光致电荷和空穴对迁移和分离行为中的作用示意图(b), CN、CNT-8、CNT-12和CNT-16的PL谱(c) 和Nyquist图(d) ${ }^{[120]}$

Figure 8 (Color online) Transient photocurrent response of CN, CNT-8, CNT-12, CNT-16 (a), schematic diagram describing the role of N-vacancies in photoinduced charges and holes on migration and separation behavior (b), PL spectra (c), and Nyquist plots of CN, CNT-8, CNT-12, and CNT-16 $(\mathrm{d})^{[120]}$

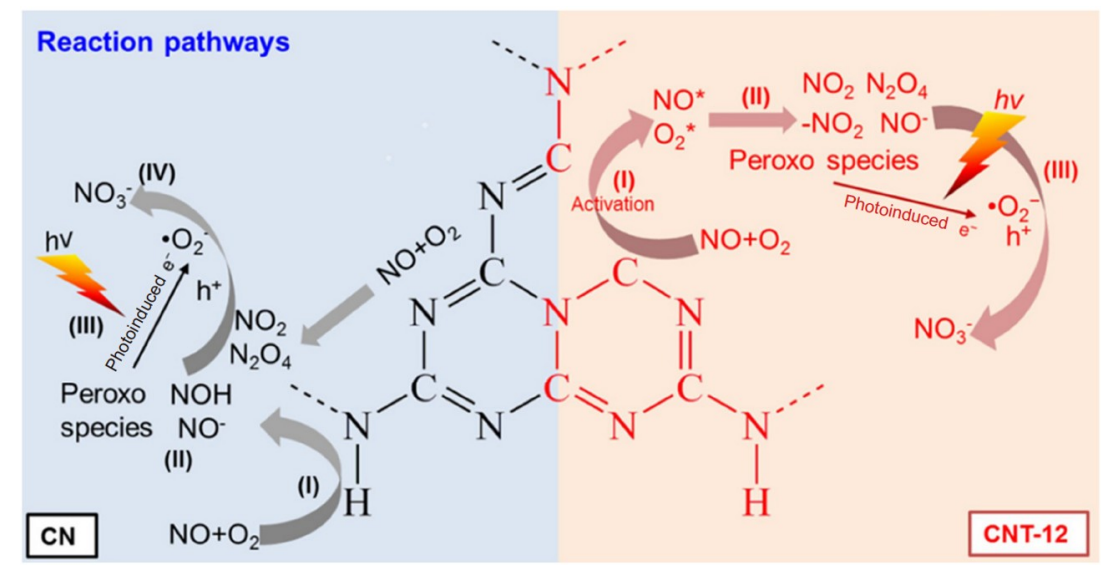

图 9 (网络版彩色)在原始(左)和缺氮(右) $g-\mathrm{C}_{3} \mathrm{~N}_{4}$ 上对 $\mathrm{NO}$ 的吸附和光催化氧化的拟反应途径 ${ }^{[120]}$

Figure 9 (Color online) Pseudo-reaction pathways for NO adsorption and photocatalytic oxidation on the original (left) and nitrogen-deficient (right)

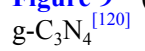


硝基化合物(- $\left.\mathrm{NO}_{2}\right)$ (图9, 右图(II)), 而不是 $\mathrm{NOH}$ 或 $\mathrm{OOH}$ 键. 辐照后, 吸收态的中间体在 $\mathrm{N}$ 空位被 $\cdot \mathrm{O}_{2}{ }^{-}$和 $\mathrm{h}^{+}$氧化 为 $\mathrm{NO}_{3}{ }^{-}$, 如图9右图(III)所示.

以上结果表明, 引人表面氮空位对 $g-\mathrm{C}_{3} \mathrm{~N}_{4}$ 的光吸 收能力增强具有积极作用, 氮空位可作为反应物吸附 活化和光诱导电子俘获的特殊位点. 此外, $\mathrm{g}-\mathrm{C}_{3} \mathrm{~N}_{4}$ 微管 的多孔结构有利于反应物的扩散, 其管状结构有利于 载流子的定向转移. 同时引人氮空位和多孔管结构, 能 够为 $\mathrm{g}-\mathrm{C}_{3} \mathrm{~N}_{4}$ 的大气污染控制提供了一种可行的策略.

\section{5 结论和展望}

本文系统地综述了缺陷 $g-\mathrm{C}_{3} \mathrm{~N}_{4}$ 的合成方法、缺陷 位点对 $\mathrm{g}-\mathrm{C}_{3} \mathrm{~N}_{4}$ 的影响以及其在水处理中的应用, 如抗 生素、有机农药的降解及降低重金属的毒性等处理方 面, 还有在水分解、二氧化碳转化及光催化脱氮上的 应用. 作为一种极具潜力的非金属光催化剂, $g-\mathrm{C}_{3} \mathrm{~N}_{4}$ 具
有很高的关注度. 截至目前, 虽然近年来已经取得了相 当大的进展，但制备具有高效催化性能的 $\mathrm{g}-\mathrm{C}_{3} \mathrm{~N}_{4}$ 仍有 诸多挑战. 因此, 要充分利用 $\mathrm{g}-\mathrm{C}_{3} \mathrm{~N}_{4}$ 在复合光催化剂中 的优异结构和电子性能, 研究还需更深人. 为了使 $\mathrm{g}-\mathrm{C}_{3} \mathrm{~N}_{4}$ 能够在市场上取得广泛应用, 进一步提高它的 光催化能力, 可以在以下几方面展开研究: (1) 将少量 的金属或非金属元素掺杂到缺陷 $g-\mathrm{C}_{3} \mathrm{~N}_{4}$ 中, 能够调整 $\mathrm{g}$ $\mathrm{C}_{3} \mathrm{~N}_{4}$ 的能带和电子结构, 对电子空穴对的分离具有促 进作用. (2) 为了使缺陷 $g-\mathrm{C}_{3} \mathrm{~N}_{4}$ 表面具有更多的活性位 点, 可优化制备方法以达到表面氮缺陷的精确控制. 同 时, 也可以进一步改变 $\mathrm{g}-\mathrm{C}_{3} \mathrm{~N}_{4}$ 的结构以增大其比表面 积, 使其催化活性更强, 达到最佳的反应效果. (3) 深人 探究光催化反应过程中氮缺陷对反应的促进机理, 研 究缺陷位点对局部电荷密度及反应活化能的影响, 这 对合理设计高效的 $g-\mathrm{C}_{3} \mathrm{~N}_{4}$ 基光催化剂具有重要理论指 导意义.

\section{参考文献}

1 Briggs D. Environmental pollution and the global burden of disease. Br Med Bull, 2003, 68: 1-24

2 Ader C R. A longitudinal study of agenda setting for the issue of environmental pollution. J Mass Commun Q, 1995, 72: 300-311

3 Wu Q Q, Qiao W, Su R. Research progress of heterogeneous photocatalysis in the fields of energy, environment and organic synthesis (in Chinese). Chin Sci Bull, 2019, 64: 3309-3326 [吴琦琪, 乔玮, 苏韧. 异相光催化技术在能源与环境及有机合成领域的研究进展. 科学通报, 2019, 64: 3309-3326]

4 Wang B, Zhao M M, Zhou Y, et al. Research progress and challenges of photocatalytic reduction of carbon dioxide to produce solar fuel (in Chinese). Sci China Tech Sci, 2017, 47: 286-296 [王冰, 赵美明, 周勇, 等. 光催化还原二氧化碳制备太阳燃料研究进展及挑战. 中国科学: 技 术科学, 2017, 47: 286-296]

5 Liang X, Chen L F, Zhang L, et al. Applications of metal-organic frameworks in photocatalysis (in Chinese). Chin Sci Bull, 2018, 63: 248-265 [梁祥, 陈莲芬, 张利, 等. 金属-有机框架在光催化中的应用. 科学通报, 2018, 63: 248-265]

6 Fujishima A, Honda K. Electrochemical photolysis of water at a semiconductor electrode. Nature, 1972, 238: 37-38

7 Yu J, Feng H, Tang L, et al. Metal-free carbon materials for persulfate-based advanced oxidation process: Microstructure, property and tailoring. Prog Mater Sci, 2020, 111: 100654

8 Feng H, Tang L, Zeng G, et al. Electron density modulation of $\mathrm{Fe}_{1-x} \mathrm{Co}_{x} \mathrm{P}$ nanosheet arrays by iron incorporation for highly efficient water splitting. Nano Energy, 2020, 67: 104174

9 Zhu X, Gao L, Tang L, et al. Ultrathin PtNi nanozyme based self-powered photoelectrochemical aptasensor for ultrasensitive chloramphenicol detection. Biosens Bioelectron, 2019, 146: 111756

10 Yu M, Wang J, Tang L, et al. Intimate coupling of photocatalysis and biodegradation for wastewater treatment: Mechanisms, recent advances and environmental applications. Water Res, 2020, 175: 115673

11 Feng H, Yu J, Tang L, et al. Tuning electron density endows $\mathrm{Fe}_{1-x} \mathrm{Co}_{x} \mathrm{P}$ with exceptional capability of electrooxidation of organic pollutants. Environ Sci Technol, 2019, 53: 13878-13887

12 Tang L, Feng H, Tang J, et al. Treatment of arsenic in acid wastewater and river sediment by $\mathrm{Fe} @ \mathrm{Fe}_{2} \mathrm{O}_{3}$ nanobunches: The effect of environmental conditions and reaction mechanism. Water Res, 2017, 117: 175-186

13 Pruden A, Ollis D F. Photoassisted heterogeneous catalysis: The degradation of trichloroethylene in water. J Catal, 1983, 82: 404-417

14 Teter D M, Hemley R J. Low-compressibility carbon nitrides. Science, 1996, 271: 53-55

15 Wang X, Maeda K, Thomas A, et al. A metal-free polymeric photocatalyst for hydrogen production from water under visible light. Nat Mater, 2009, 8: 76-80 
16 Ong W J, Tan L L, Ng Y H, et al. Graphitic carbon nitride $\left(\mathrm{g}^{-} \mathrm{C}_{3} \mathrm{~N}_{4}\right)$-based photocatalysts for artificial photosynthesis and environmental remediation: Are we a step closer to achieving sustainability? Chem Rev, 2016, 116: 7159-7329

17 Li X, Yu J, Low J, et al. Engineering heterogeneous semiconductors for solar water splitting. J Mater Chem A, 2015, 3: 2485-2534

18 Chen X, Shen S, Guo L, et al. Semiconductor-based photocatalytic hydrogen generation. Chem Rev, 2010, 110: 6503-6570

19 Chen C, Ma W, Zhao J. Semiconductor-mediated photodegradation of pollutants under visible-light irradiation. Chem Soc Rev, 2010, 39: 42064219

20 Kudo A, Miseki Y. Heterogeneous photocatalyst materials for water splitting. Chem Soc Rev, 2009, 38: 253-278

21 Bard A J, Fox M A. Artificial photosynthesis: Solar splitting of water to hydrogen and oxygen. Acc Chem Res, 1995, 28: 141-145

22 Malato S, Fernández-Ibáñez P, Maldonado M I, et al. Decontamination and disinfection of water by solar photocatalysis: Recent overview and trends. Catal Today, 2009, 147: 1-59

23 Cui Y, Ding Z, Fu X, et al. Construction of conjugated carbon nitride nanoarchitectures in solution at low temperatures for photoredox catalysis. Angew Chem Int Ed, 2012, 51: 11814-11818

24 Wang Y, Li H, Yao J, et al. Synthesis of boron doped polymeric carbon nitride solids and their use as metal-free catalysts for aliphatic C-H bond oxidation. Chem Sci, 2011, 2: 446-450

25 Shiraishi Y, Hirai T. Selective organic transformations on titanium oxide-based photocatalysts. J Photochem Photobiol C-Photochem Rev, 2008, 9: $157-170$

26 Kirsch P. Modern Fluoroorganic Chemistry: Synthesis, Reactivity, Applications. New Jersey: John Wiley \& Sons, 2013

27 Lang X, Chen X, Zhao J. Heterogeneous visible light photocatalysis for selective organic transformations. Chem Soc Rev, 2014, 43: 473-486

28 Wang Y, Zhang J, Wang X, et al. Boron- and fluorine-containing mesoporous carbon nitride polymers: Metal-free catalysts for cyclohexane oxidation. Angew Chem Int Ed, 2010, 49: 3356-3359

29 Goettmann F, Thomas A, Antonietti M. Metal-free activation of $\mathrm{CO}_{2}$ by mesoporous graphitic carbon nitride. Angew Chem Int Ed, 2007, 46: $2717-2720$

30 Li X, Wen J, Low J, et al. Design and fabrication of semiconductor photocatalyst for photocatalytic reduction of $\mathrm{CO}_{2}$ to solar fuel. Sci China Mater, 2014, 57: 70-100

31 Low J, Yu J, Ho W. Graphene-based photocatalysts for $\mathrm{CO}_{2}$ reduction to solar fuel. J Phys Chem Lett, 2015, 6: 4244-4251

32 Gao G, Jiao Y, Waclawik E R, et al. Single atom (Pd/Pt) supported on graphitic carbon nitride as an efficient photocatalyst for visible-light reduction of carbon dioxide. J Am Chem Soc, 2016, 138: 6292-6297

33 Marszewski M, Cao S, Yu J, et al. Semiconductor-based photocatalytic $\mathrm{CO}_{2}$ conversion. Mater Horiz, 2015, 2: 261-278

34 Park H, Park Y, Kim W, et al. Surface modification of $\mathrm{TiO}_{2}$ photocatalyst for environmental applications. J Photochem Photobiol C-Photochem Rev, 2013, 15: 1-20

35 Hoffmann M R, Martin S T, Choi W, et al. Environmental applications of semiconductor photocatalysis. Chem Rev, 1995, 95: 69-96

36 Zhang H, Chen G, Bahnemann D W. Photoelectrocatalytic materials for environmental applications. J Mater Chem, 2009, 19: 5089-5121

37 Pelaez M, Nolan N T, Pillai S C, et al. A review on the visible light active titanium dioxide photocatalysts for environmental applications. Appl Catal B-Environ, 2012, 125: 331-349

38 Wang H, Yuan X, Wu Y, et al. Photodeposition of metal sulfides on titanium metal-organic frameworks for excellent visible-light-driven photocatalytic $\mathrm{Cr}(\mathrm{Vi})$ reduction. RSC Adv, 2015, 5: 32531-32535

39 Chong M N, Jin B, Chow C W K, et al. Recent developments in photocatalytic water treatment technology: A review. Water Res, 2010, 44: 29973027

40 Liu G, Zhen C, Kang Y, et al. Unique physicochemical properties of two-dimensional light absorbers facilitating photocatalysis. Chem Soc Rev, 2018, 47: 6410-6444

41 Lau V W, Mesch M B, Duppel V, et al. Low-molecular-weight carbon nitrides for solar hydrogen evolution. J Am Chem Soc, 2015, 137: 10641072

42 Dong G, Zhang L. Porous structure dependent photoreactivity of graphitic carbon nitride under visible light. J Mater Chem, 2012, 22: 1160-1166

43 Deng Y, Tang L, Zeng G, et al. Insight into highly efficient simultaneous photocatalytic removal of Cr(VI) and 2,4-diclorophenol under visible light irradiation by phosphorus doped porous ultrathin $\mathrm{g}-\mathrm{C}_{3} \mathrm{~N}_{4}$ nanosheets from aqueous media: Performance and reaction mechanism. Appl Catal B-Environ, 2017, 203: 343-354

44 Dong G, Zhao K, Zhang L. Carbon self-doping induced high electronic conductivity and photoreactivity of g-C $\mathrm{N}_{3}$. Chem Commun, 2012, 48: $6178-6180$

45 Yan S C, Li Z S, Zou Z G. Photodegradation of rhodamine B and methyl orange over boron-doped g- $\mathrm{C}_{3} \mathrm{~N}_{4}$ under visible light irradiation. Langmuir, 2010, 26: 3894-3901

46 Wei F, Liu Y, Zhao $\mathrm{H}$, et al. Oxygen self-doped $\mathrm{g}-\mathrm{C}_{3} \mathrm{~N}_{4}$ with tunable electronic band structure for unprecedentedly enhanced photocatalytic 
performance. Nanoscale, 2018, 10: 4515-4522

47 Liu G, Niu P, Sun C, et al. Unique electronic structure induced high photoreactivity of sulfur-doped graphitic $\mathrm{C}_{3} \mathrm{~N}_{4}$. J Am Chem Soc, 2010, 132: 11642-11648

48 Zhang Y, Mori T, Ye J, et al. Phosphorus-doped carbon nitride solid: Enhanced electrical conductivity and photocurrent generation. J Am Chem Soc, 2010, 132: 6294-6295

49 Lan Z A, Zhang G, Wang X. A facile synthesis of Br-modified g- $\mathrm{C}_{3} \mathrm{~N}_{4}$ semiconductors for photoredox water splitting. Appl Catal B-Environ, 2016, 192: 116-125

50 Chen X, Zhang J, Fu X, et al. Fe-g- $\mathrm{C}_{3} \mathrm{~N}_{4}$-catalyzed oxidation of benzene to phenol using hydrogen peroxide and visible light. J Am Chem Soc, 2009, 131: 11658-11659

51 Ge L, Han C, Liu J. Novel visible light-induced g- $\mathrm{C}_{3} \mathrm{~N}_{4} / \mathrm{Bi}_{2} \mathrm{WO}_{6}$ composite photocatalysts for efficient degradation of methyl orange. Appl Catal B-Environ, 2011, 108-109: 100-107

$52 \mathrm{Xu} \mathrm{H}$, Yan J, Xu Y, et al. Novel visible-light-driven $\mathrm{AgX} /$ graphite-like $\mathrm{C}_{3} \mathrm{~N}_{4}(\mathrm{X}=\mathrm{Br}$, I) hybrid materials with synergistic photocatalytic activity. Appl Catal B-Environ, 2013, 129: 182-193

53 Fu J, Chang B, Tian Y, et al. Novel $\mathrm{C}_{3} \mathrm{~N}_{4}$-CdS composite photocatalysts with organic-inorganic heterojunctions: In situ synthesis, exceptional activity, high stability and photocatalytic mechanism. J Mater Chem A, 2013, 1: 3083-3090

54 Han $\mathrm{C}$, Wang $\mathrm{Y}$, Lei $\mathrm{Y}$, et al. In situ synthesis of graphitic- $\mathrm{C}_{3} \mathrm{~N}_{4}$ nanosheet hybridized $\mathrm{N}$-doped $\mathrm{TiO}_{2}$ nanofibers for efficient photocatalytic $\mathrm{H}_{2}$ production and degradation. Nano Res, 2015, 8: 1199-1209

55 Kumar S, Surendar T, Baruah A, et al. Synthesis of a novel and stable $\mathrm{g}^{-} \mathrm{C}_{3} \mathrm{~N}_{4}-\mathrm{Ag}_{3} \mathrm{PO}_{4}$ hybrid nanocomposite photocatalyst and study of the photocatalytic activity under visible light irradiation. J Mater Chem A, 2013, 1: 5333-5340

56 Ge L, Han C. Synthesis of MWNTs $/ \mathrm{g}-\mathrm{C}_{3} \mathrm{~N}_{4}$ composite photocatalysts with efficient visible light photocatalytic hydrogen evolution activity. Appl Catal B-Environ, 2012, 117-118: 268-274

57 Ge L, Han C, Liu J. In situ synthesis and enhanced visible light photocatalytic activities of novel PANI-g- $\mathrm{C}_{3} \mathrm{~N}_{4}$ composite photocatalysts. J Mater Chem, 2012, 22: 11843-11850

58 Du A, Sanvito S, Li Z, et al. Hybrid graphene and graphitic carbon nitride nanocomposite: Gap opening, electron-hole puddle, interfacial charge transfer, and enhanced visible light response. J Am Chem Soc, 2012, 134: 4393-4397

59 Deng Y, Tang L, Feng C, et al. Construction of plasmonic Ag and nitrogen-doped graphene quantum dots codecorated ultrathin graphitic carbon nitride nanosheet composites with enhanced photocatalytic activity: Full-spectrum response ability and mechanism insight. ACS Appl Mater Interfaces, 2017, 9: 42816-42828

60 Niu P, Liu G, Cheng H M. Nitrogen vacancy-promoted photocatalytic activity of graphitic carbon nitride. J Phys Chem C, 2012, 116: 1101311018

61 Molina B, Sansores L E. Electronic structure of six phases of $\mathrm{C}_{3} \mathrm{~N}_{4}$ : A theoretical approach. Mod Phys Lett B, 1999, 13: 193-201

62 Kroke E, Schwarz M, Horath-Bordon E, et al. Tri-s-triazine derivatives. Part I. From trichloro-tri-s-triazine to graphitic $\mathrm{C}_{3} \mathrm{~N}_{4}$ structures. New J Chem, 2002, 26: 508-512

$63 \mathrm{Xu} \mathrm{Y,} \mathrm{Gao} \mathrm{S} \mathrm{P.} \mathrm{Band} \mathrm{gap} \mathrm{of} \mathrm{C}_{3} \mathrm{~N}_{4}$ in the GW approximation. Int J Hydrog Energy, 2012, 37: 11072-11080

64 Jürgens B, Irran E, Senker J, et al. Melem (2,5,8-triamino-tri-s-triazine), an important intermediate during condensation of melamine rings to graphitic carbon nitride: Synthesis, structure determination by X-ray powder diffractometry, solid-state NMR, and theoretical studies. J Am Chem Soc, 2003, 125: 10288-10300

65 Sehnert J, Baerwinkel K, Senker J. Ab initio calculation of solid-state NMR spectra for different triazine and heptazine based structure proposals of g- $\mathrm{C}_{3} \mathrm{~N}_{4}$. J Phys Chem B, 2007, 111: 10671-10680

66 Zhang J S, Wang B, Wang X C. Chemical synthesis and application of graphite carbon nitride (in Chinese). J Phys Chem, 2013, 29: 1865-1876 [张金水, 王博, 王心晨. 石墨相氮化碳的化学合成及应用. 物理化学学报, 2013, 29: 1865-1876]

67 Bojdys M J, Müller J O, Antonietti M, et al. Ionothermal synthesis of crystalline, condensed, graphitic carbon nitride. Chem-A Eur J, 2008, 14: $8177-8182$

68 McAllister M J, Li J L, Adamson D H, et al. Single sheet functionalized graphene by oxidation and thermal expansion of graphite. Chem Mater, 2007, 19: 4396-4404

69 Wen J, Xie J, Chen X, et al. A review on $\mathrm{g}_{-} \mathrm{C}_{3} \mathrm{~N}_{4}$-based photocatalysts. Appl Surf Sci, 2017, 391: 72-123

70 Li Y, Zhang J, Wang Q, et al. Nitrogen-rich carbon nitride hollow vessels: Synthesis, characterization, and their properties. J Phys Chem B, 2010, 114: 9429-9434

71 Komatsu T. Prototype carbon nitrides similar to the symmetric triangular form of melon. J Mater Chem, 2001, 11: 802-803

72 Haque E, Jun J W, Talapaneni S N, et al. Superior adsorption capacity of mesoporous carbon nitride with basic CN framework for phenol. J Mater Chem, 2010, 20: 10801-10803 
73 Zhou L, Xu Y, Yu W, et al. Ultrathin two-dimensional graphitic carbon nitride as a solution-processed cathode interfacial layer for inverted polymer solar cells. J Mater Chem A, 2016, 4: 8000-8004

74 Xue F, Sun J, Shen T T, et al. Improvement strategy based on defect design of $\mathrm{g}-\mathrm{C}_{3} \mathrm{~N}_{4}$ photocatalyst (in Chinese). J Qilu Univ Technol, 2019, 33: 44-50 [薛芳, 孙静, 申婷婷, 等. 基于 $\mathrm{g}_{-} \mathrm{C}_{3} \mathrm{~N}_{4}$ 光催化剂缺陷设计的改进策略. 齐鲁工业大学学报, 2019, 33: 44-50]

75 Zhang S, Gu P, Ma R, et al. Recent developments in fabrication and structure regulation of visible-light-driven g- $\mathrm{C}_{3} \mathrm{~N}_{4}$-based photocatalysts towards water purification: A critical review. Catal Today, 2019, 335: 65-77

76 Jiang W, Luo W, Wang J, et al. Enhancement of catalytic activity and oxidative ability for graphitic carbon nitride. J Photochem Photobiol CPhotochem Rev, 2016, 28: 87-115

$77 \mathrm{Yu} \mathrm{W}$, Chen J, Shang T, et al. Direct Z-scheme g- $\mathrm{C}_{3} \mathrm{~N}_{4} / \mathrm{WO}_{3}$ photocatalyst with atomically defined junction for $\mathrm{H}_{2}$ production. Appl Catal BEnviron, 2017, 219: 693-704

78 Nowotny M K, Sheppard L R, Bak T, et al. Defect chemistry of titanium dioxide. Application of defect engineering in processing of TiO ${ }_{2}$-based photocatalysts. J Phys Chem C, 2008, 112: 5275-5300

79 Gong X Q, Selloni A, Batzill M, et al. Steps on anatase $\mathrm{TiO}_{2}(101)$. Nat Mater, 2006, 5: 665-670

80 Thompson T L, Yates J T. Surface science studies of the photoactivation of $\mathrm{TiO}_{2}$ new photochemical processes. Chem Rev, 2006, 106: 4428-4453

81 Suriye K, Praserthdam P, Jongsomjit B. Control of $\mathrm{Ti}^{3+}$ surface defect on $\mathrm{TiO}_{2}$ nanocrystal using various calcination atmospheres as the first step for surface defect creation and its application in photocatalysis. Appl Surf Sci, 2007, 253: 3849-3855

82 Chen X, Liu L, Yu P Y, et al. Increasing solar absorption for photocatalysis with black hydrogenated titanium dioxide nanocrystals. Science, 2011, 331: 746-750

$83 \mathrm{Xu} \mathrm{C} \mathrm{Q}$, Li K, Zhang W D. Enhancing visible light photocatalytic activity of nitrogen-deficient $\mathrm{g}-\mathrm{C}_{3} \mathrm{~N}_{4}$ via thermal polymerization of acetic acidtreated melamine. J Colloid Interface Sci, 2017, 495: 27-36

$84 \mathrm{Yu} \mathrm{H}$, Shi R, Zhao Y, et al. Alkali-assisted synthesis of nitrogen deficient graphitic carbon nitride with tunable band structures for efficient visiblelight-driven hydrogen evolution. Adv Mater, 2017, 29: 1605148

85 Wang Y, Zhao S, Zhang Y, et al. Facile synthesis of self-assembled g- $\mathrm{C}_{3} \mathrm{~N}_{4}$ with abundant nitrogen defects for photocatalytic hydrogen evolution. ACS Sustain Chem Eng, 2018, 6: 10200-10210

86 Gao H, Yan S, Wang J, et al. Towards efficient solar hydrogen production by intercalated carbon nitride photocatalyst. Phys Chem Chem Phys, 2013, 15: 18077-18084

87 Wang D, Xu Z, Luo Q, et al. Preparation and visible-light photocatalytic performances of $\mathrm{g}_{-} \mathrm{C}_{3} \mathrm{~N}_{4}$ surface hybridized with a small amount of CdS nanoparticles. J Mater Sci, 2016, 51: 893-902

88 Cui Y, Zhang G, Lin Z, et al. Condensed and low-defected graphitic carbon nitride with enhanced photocatalytic hydrogen evolution under visible light irradiation. Appl Catal B-Environ, 2016, 181: 413-419

$89 \mathrm{Tu} \mathrm{W}, \mathrm{Xu} \mathrm{Y}$, Wang J, et al. Investigating the role of tunable nitrogen vacancies in graphitic carbon nitride nanosheets for efficient visible-lightdriven $\mathrm{H}_{2}$ evolution and $\mathrm{CO}_{2}$ reduction. ACS Sustain Chem Eng, 2017, 5: 7260-7268

90 Zhang G, Li G, Lan Z A, et al. Optimizing optical absorption, exciton dissociation, and charge transfer of a polymeric carbon nitride with ultrahigh solar hydrogen production activity. Angew Chem Int Ed, 2017, 56: 13445-13449

91 Liu J, Fang W, Wei Z, et al. Efficient photocatalytic hydrogen evolution on N-deficient g- $\mathrm{C}_{3} \mathrm{~N}_{4}$ achieved by a molten salt post-treatment approach Appl Catal B-Environ, 2018, 238: 465-470

92 Liang L, Shi L, Wang F. Fabrication of large surface area nitrogen vacancy modified graphitic carbon nitride with improved visible-light photocatalytic performance. Diam Relat Mat, 2019, 91: 230-236

93 Chen J, Mao Z, Zhang L, et al. Nitrogen-deficient graphitic carbon nitride with enhanced performance for lithium ion battery anodes. ACS Nano, 2017, 11: 12650-12657

$94 \mathrm{Ma} \mathrm{H}$, Shi Z, Li Q, et al. Preparation of graphitic carbon nitride with large specific surface area and outstanding $\mathrm{N}_{2}$ photofixation ability via a dissolve-regrowth process. J Phys Chem Solids, 2016, 99: 51-58

95 Li X, Yu J, Jaroniec M. Hierarchical photocatalysts. Chem Soc Rev, 2016, 45: 2603-2636

96 Xie Y, Li Y, Huang Z, et al. Two types of cooperative nitrogen vacancies in polymeric carbon nitride for efficient solar-driven $\mathrm{H}_{2} \mathrm{O}_{2}$ evolution Appl Catal B-Environ, 2020, 265: 118581

97 Zhang Y, Gao J, Chen Z. A solid-state chemical reduction approach to synthesize graphitic carbon nitride with tunable nitrogen defects for efficient visible-light photocatalytic hydrogen evolution. J Colloid Interface Sci, 2019, 535: 331-340

98 Niu P, Yin L C, Yang Y Q, et al. Increasing the visible light absorption of graphitic carbon nitride (Melon) photocatalysts by homogeneous selfmodification with nitrogen vacancies. Adv Mater, 2014, 26: 8046-8052

99 Li J, Shen B, Hong Z, et al. A facile approach to synthesize novel oxygen-doped $\mathrm{g}-\mathrm{C}_{3} \mathrm{~N}_{4}$ with superior visible-light photoreactivity. Chem Commun, 2012, 48: 12017-12019 
100 Shi W, Shu K, Sun H, et al. Dual enhancement of capturing photogenerated electrons by loading CoP nanoparticles on N-deficient graphitic carbon nitride for efficient photocatalytic degradation of tetracycline under visible light. Sep Purif Technol, 2020, 246: 116930

101 Wang $\mathrm{K}, \mathrm{Fu} \mathrm{J}$, Zheng Y. Insights into photocatalytic $\mathrm{CO}_{2}$ reduction on $\mathrm{C}_{3} \mathrm{~N}_{4}$ : Strategy of simultaneous B, K co-doping and enhancement by $\mathrm{N}$ vacancies. Appl Catal B-Environ, 2019, 254: 270-282

102 Liu X, Li C, Zhang Y, et al. Simultaneous photodegradation of multi-herbicides by oxidized carbon nitride: Performance and practical application. Appl Catal B-Environ, 2017, 219: 194-199

103 Guan W, Long Z, Liu J, et al. Unique graphitic carbon nitride nanovessels as recyclable adsorbent for solid phase extraction of benzoylurea pesticides in juices samples. Food Anal Methods, 2015, 8: 2202-2210

104 Wei K, Li K, Yan L, et al. One-step fabrication of $\mathrm{g}-\mathrm{C}_{3} \mathrm{~N}_{4}$ nanosheets $/ \mathrm{TiO}_{2}$ hollow microspheres heterojunctions with atomic level hybridization and their application in the multi-component synergistic photocatalytic systems. Appl Catal B-Environ, 2018, 222: 88-98

105 Zhang Y, Zhang Q, Shi Q, et al. Acid-treated g- $\mathrm{C}_{3} \mathrm{~N}_{4}$ with improved photocatalytic performance in the reduction of aqueous $\mathrm{Cr}(\mathrm{VI}) \mathrm{under}$ visiblelight. Sep Purif Technol, 2015, 142: 251-257

106 Guo S, Deng Z, Li M, et al. Phosphorus-doped carbon nitride tubes with a layered micro-nanostructure for enhanced visible-light photocatalytic hydrogen evolution. Angew Chem Int Ed, 2016, 55: 1830-1834

107 Ran J, Ma T Y, Gao G, et al. Porous P-doped graphitic carbon nitride nanosheets for synergistically enhanced visible-light photocatalytic $\mathrm{H}_{2}$ production. Energy Environ Sci, 2015, 8: 3708-3717

108 Kang Y, Yang Y, Yin L C, et al. An amorphous carbon nitride photocatalyst with greatly extended visible-light-responsive range for photocatalytic hydrogen generation. Adv Mater, 2015, 27: 4572-4577

109 Niu P, Qiao M, Li Y, et al. Distinctive defects engineering in graphitic carbon nitride for greatly extended visible light photocatalytic hydrogen evolution. Nano Energy, 2018, 44: 73-81

110 Wang Y, Bayazit M K, Moniz S J A, et al. Linker-controlled polymeric photocatalyst for highly efficient hydrogen evolution from water. Energy Environ Sci, 2017, 10: 1643-1651

111 Huang Y, Liu J, Zhao C, et al. Facile synthesis of defect-modified thin-layered and porous $\mathrm{g}_{-} \mathrm{C}_{3} \mathrm{~N}_{4}$ with synergetic improvement for photocatalytic $\mathrm{H}_{2}$ production. ACS Appl Mater Inter, 2020, 47: 52603-52614

112 Tang J Y, Kong X Y, Ng B J, et al. Midgap-state-mediated two-step photoexcitation in nitrogen defect-modified g- $\mathrm{C}_{3} \mathrm{~N}_{4}$ atomic layers for superior photocatalytic $\mathrm{CO}_{2}$ reduction. Catal Sci Technol, 2019, 9: 2335-2343

113 Zhang G, Zhang M, Ye X, et al. Iodine modified carbon nitride semiconductors as visible light photocatalysts for hydrogen evolution. Adv Mater, 2014, 26: 805-809

114 Bai S, Wang X, Hu C, et al. Two-dimensional g- $\mathrm{C}_{3} \mathrm{~N}_{4}$ : An ideal platform for examining facet selectivity of metal co-catalysts in photocatalysis. Chem Commun, 2014, 50: 6094-6097

115 Zhou S, Liu Y, Li J, et al. Facile in situ synthesis of graphitic carbon nitride $\left(\mathrm{g}-\mathrm{C}_{3} \mathrm{~N}_{4}\right)-\mathrm{N}-\mathrm{TiO}_{2}$ heterojunction as an efficient photocatalyst for the selective photoreduction of $\mathrm{CO}_{2}$ to $\mathrm{CO}$. Appl Catal B-Environ, 2014, 158-159: 20-29

$116 \mathrm{Li} \mathrm{M}$, Zhang L, Fan X, et al. Highly selective $\mathrm{CO}_{2}$ photoreduction to $\mathrm{CO}$ over $\mathrm{g}-\mathrm{C}_{3} \mathrm{~N}_{4} / \mathrm{Bi}_{2} \mathrm{WO}_{6}$ composites under visible light. J Mater Chem A, 2015, 3: 5189-5196

117 Wang J C, Yao H C, Fan Z Y, et al. Indirect Z-scheme BiOI/g- $\mathrm{C}_{3} \mathrm{~N}_{4}$ photocatalysts with enhanced photoreduction $\mathrm{CO}_{2}$ activity under visible light irradiation. ACS Appl Mater Interfaces, 2016, 8: 3765-3775

$118 \mathrm{Li} \mathrm{M}$, Zhang L, Wu M, et al. Mesostructured $\mathrm{CeO}_{2} / \mathrm{g}-\mathrm{C}_{3} \mathrm{~N}_{4}$ nanocomposites: Remarkably enhanced photocatalytic activity for $\mathrm{CO}_{2}$ reduction by mutual component activations. Nano Energy, 2016, 19: 145-155

$119 \mathrm{Li} \mathrm{M}$, Zhang L, Fan X, et al. Core-shell $\mathrm{LaPO}_{4} / \mathrm{g}-\mathrm{C}_{3} \mathrm{~N}_{4}$ nanowires for highly active and selective $\mathrm{CO}_{2}$ reduction. Appl Catal B-Environ, 2017, 201: 629-635

120 Wang Z, Huang Y, Chen M, et al. Roles of N-vacancies over porous $\mathrm{g}^{-} \mathrm{C}_{3} \mathrm{~N}_{4}$ microtubes during photocatalytic $\mathrm{NO}_{x}$ removal. ACS Appl Mater Interfaces, 2019, 11: 10651-10662

\section{补充材料}

表 $\mathrm{S} 1$ 缺陷 $\mathrm{g}-\mathrm{C}_{3} \mathrm{~N}_{4}$ 不同制备方法及其产氢速率的比较

表 S2 缺陷氮化碳(CN-400)与其他 $\mathrm{g}-\mathrm{C}_{3} \mathrm{~N}_{4}$ 光催化剂的 $\mathrm{H}_{2}$ 生成速率比较

表S3 CN-525与其他氮化碳催化剂将 $\mathrm{CO}_{2}$ 还原为 $\mathrm{CO}$ 的活性比较

本文以上补充材料见网络版csb.scichina.com. 补充材料为作者提供的原始数据, 作者对其学术质量和内容负责. 


\title{
Preparation and application of defective graphite phase carbon nitride photocatalysts
}

\author{
Shanshan $\mathrm{Ye}^{1,2 \dagger}$, Chengyang Feng ${ }^{1,2 \dagger}$, Jiajia Wang ${ }^{1,2^{*}} \&$ Lin Tang $^{1,2^{*}}$ \\ ${ }^{1}$ College of Environmental Science and Engineering, Hunan University, Changsha 410082, China; \\ ${ }^{2}$ Key Laboratory of Environmental Biology and Pollution Control (Hunan University), Ministry of Education, Changsha 410082, China \\ $\uparrow$ Equally contributed to this work \\ * Corresponding authors, E-mail: wangjiajia07@hnu.edu.cn; tanglin@hnu.edu.cn
}

With the development of industry and agriculture, the problems of environmental pollution and energy shortage have become increasingly severe. Semiconductor photocatalysis technology is one of the effective ways to solve environmental pollution and energy crisis. The principle of photocatalysis is based on the oxidation-reduction ability of photocatalysts under light conditions, which can achieve the purposes of purification of pollutants, material synthesis and transformation. Graphite phase carbon nitride $\left(\mathrm{g}-\mathrm{C}_{3} \mathrm{~N}_{4}\right)$, as a new high-efficiency catalyst, has good stability and shows great engineering application potential in photocatalytic technology. However, the unmodified $\mathrm{g}_{-} \mathrm{C}_{3} \mathrm{~N}_{4}$ has a limited visible light response range, and the photo-excited charge carrier recombination rate is high, resulting in low photocatalytic activity. Nitrogen defects are introduced into the $\mathrm{g}-\mathrm{C}_{3} \mathrm{~N}_{4}$ framework. These defects can manipulate the electronic structure, and the interstitial state produced can be used as a band-tail state, which can overlap with the valence band or the conduction band. The midgap state of semiconductors can extend the light response and act as an active center for electron-hole excitation. Introducing defects into g- $\mathrm{C}_{3} \mathrm{~N}_{4}$ can improve the photocatalytic activity of g- $\mathrm{C}_{3} \mathrm{~N}_{4}$.

This paper systematically reviews the physical, chemical and electrochemical properties of $\mathrm{g}_{-} \mathrm{C}_{3} \mathrm{~N}_{4}$ on the basis of experimental and theoretical research progress. The synthesis methods of defect $\mathrm{g}-\mathrm{C}_{3} \mathrm{~N}_{4}$ are summarized, including adjustment before polymerization, adjustment during polymerization, and adjustment after polymerization. The adjustment before polymerization is to introduce defects by changing the precursor, such as adding hydroxide, sodium borohydride and other substances to the precursor. The adjustment during polymerization is to provide a reducing atmosphere during polymerization can prepare $\mathrm{g}_{-} \mathrm{C}_{3} \mathrm{~N}_{4}$ with different nitrogen-vacancy densities, such as hydrogen, ammonia and so on. The adjustment after polymerization is to modify the synthesized defect-free $\mathrm{g}_{-} \mathrm{C}_{3} \mathrm{~N}_{4}$, such as recalcining or acid treatment to achieve the purpose of synthesizing nitrogen vacancies. The effect of defect sites on $\mathrm{g}-\mathrm{C}_{3} \mathrm{~N}_{4}$ is also discussed. The intermediate state induced by nitrogen defects can be transformed into active centers excited by electron holes, and the optical response of defect $\mathrm{g}_{-} \mathrm{C}_{3} \mathrm{~N}_{4}$ is broadened due to the narrowing of the band gap. In the range, nitrogen defects can trap photo-generated carriers and prevent their recombination, thereby increasing the overall quantum efficiency. However, excessive introduction of nitrogen defects will produce deeper interstitial states. These deeper interstitial states can not only capture photo-generated electrons, but also photo-generated $\mathrm{h}^{+}$, which then become the recombination sites of photogenerated carriers. In addition, we separately summarized the application of the defect $g-\mathrm{C}_{3} \mathrm{~N}_{4}$ in water treatment, such as the degradation of antibiotics and organic pesticides and the reduction of the toxicity of heavy metals, as well as water decomposition, carbon dioxide conversion and photocatalytic denitrification. Defect $\mathrm{g}-\mathrm{C}_{3} \mathrm{~N}_{4}$ has achieved good results in these applications. Although considerable progress has been made in the research of ${ }_{-}-\mathrm{C}_{3} \mathrm{~N}_{4}$ in recent years, there are still many challenges in preparing g- $\mathrm{C}_{3} \mathrm{~N}_{4}$ with high-efficiency catalytic performance. Finally, in view of the challenges faced by the application of defective $\mathrm{g}-\mathrm{C}_{3} \mathrm{~N}_{4}$, key discussions and future prospects are proposed from the aspects of mechanism exploration and material development.

g- $\mathrm{C}_{3} \mathrm{~N}_{4}$, nitrogen defects, photocatalysis, electron-hole pair, band structure regulation

doi: 10.1360/TB-2020-1674 